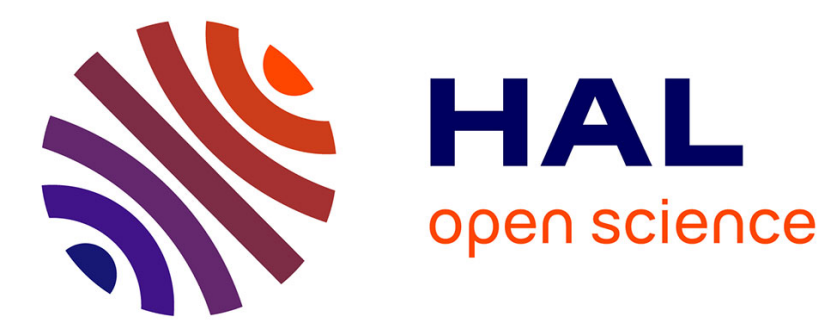

\title{
Coupling Boltzmann and Navier-Stokes Equations by Friction
}

\author{
Jean-François Bourgat, Patrick Le Tallec, Moulay D. Tidriri
}

\section{To cite this version:}

Jean-François Bourgat, Patrick Le Tallec, Moulay D. Tidriri. Coupling Boltzmann and Navier-Stokes

Equations by Friction. [Research Report] RR-2483, INRIA. 1995. inria-00074192

\section{HAL Id: inria-00074192 \\ https://hal.inria.fr/inria-00074192}

Submitted on 24 May 2006

HAL is a multi-disciplinary open access archive for the deposit and dissemination of scientific research documents, whether they are published or not. The documents may come from teaching and research institutions in France or abroad, or from public or private research centers.
L'archive ouverte pluridisciplinaire HAL, est destinée au dépôt et à la diffusion de documents scientifiques de niveau recherche, publiés ou non, émanant des établissements d'enseignement et de recherche français ou étrangers, des laboratoires publics ou privés. 
INSTITUT NATIONAL DE RECHERCHE EN INFORMATIQUE ET EN AUTOMATIQUE

\title{
Coupling Boltzmann and Navier-Stokes Equations by Friction.
}

\author{
Jean-François Bourgat, Patrick Le Tallec , Moulay D. Tidriri
}

\section{$\mathbf{N}^{\circ} 2483$}

February 1995

PROGRAMME 6

Calcul scientifique, modélisation

et logiciel numérique

\section{apport}

derecherche 



\title{
Coupling Boltzmann and Navier-Stokes Equations by Friction.
}

\author{
Jean-François Bourgat *, Patrick Le Tallec ${ }^{* *}$, Moulay D. Tidriri *** \\ Programme 6 - Calcul scientifique, modélisation et logiciel numérique \\ Projet MENUSIN
}

Rapport de recherche $\mathrm{n}^{\circ} 2483$ - February $1995-27$ pages

\begin{abstract}
The aim of this paper is to introduce and validate a coupled Navier-Stokes Boltzmann approach for the calculation of hypersonic rarefied flows around manoeuvering vehicles. The proposed strategy uses locally a kinetic model in the boundary layer coupled through wall friction forces to a global Navier-Stokes solver. Different numerical experiments illustrate the potentialities of the method.
\end{abstract}

Key-words: Boltzmann, coupling algorithms, domain decomposition, diatomic gases, friction, Navier-Stokes, time marching algorithms.

(Résumé : tsvp)

${ }^{*}$ INRIA, Domaine de Voluceau- Rocquencourt- B.P. 105- Le Chesnay Cedex (France) email: JeanFrancois.Bourgat@inria.fr

${ }^{* *}$ Université Paris-Dauphine and INRIA, Domaine de Voluceau- Rocquencourt- B.P. 105- Le Chesnay Cedex (France) email: Patrick.LeTallec@inria.fr

***ICASE, Mail Stop 132C, Hampton, VA, 23 681-0001 email: tidriri@icase.edu. This work has been done in 1991 at INRIA and has been supported by the Hermes Research program under grant number RDAN 86.1/3. Special thanks are also due to F. Mallinger and B. Mohammadi for their important contribution to the numerical tests. Finally, partial support of CEA-CESTA is gratefully acknowledged. 


\section{Couplage des Equations de Boltzmann et de Navier-Stokes par frottement.}

Résumé : Le but de ce rapport est d'introduire et valider une technique de couplage des Equations de Navier-Stokes avec les Equations de Boltzmann afin de calculer les écoulements hypersoniques autour d'engins manoeuvrant à haute altitude. La stratégie proposée utilise localement un modèle cinétique en couche limite, couplé à un solveur global NavierStokes par le biais de conditions de frottement imposées à la paroi. Différentes simulations numériques illustrent les possibilités de l'approche proposée.

Mots-clé : algorithmes de couplage, Boltzmann, décomposition de domaines, frottement, gaz diatomique, marche en temps, Navier-Stokes. 


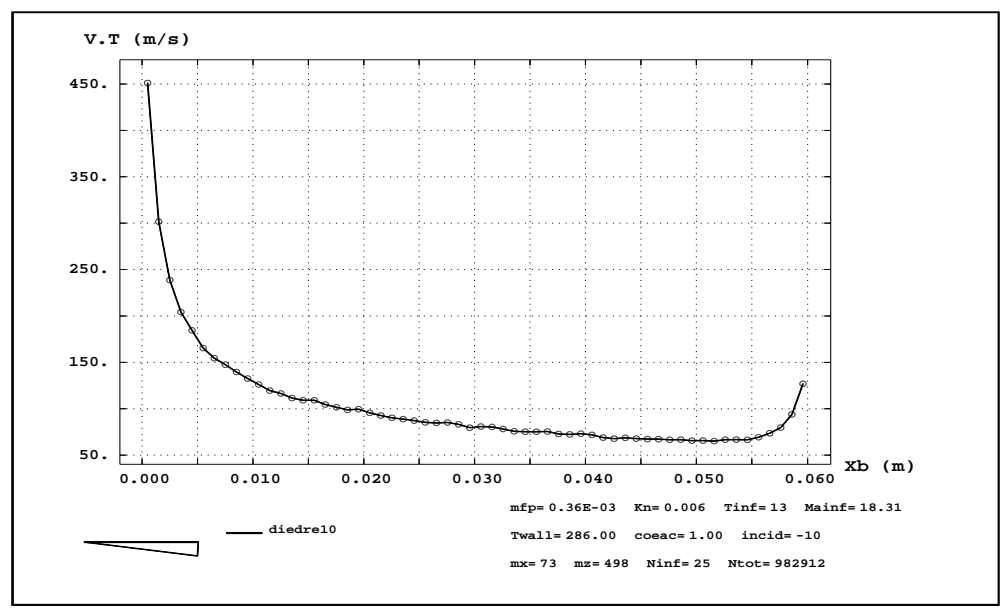

Figure 1: Slip velocity on a flate plate as predicted by a kinetic simulation

\section{Introduction}

Computing flows around manoeuvering vehicles at high altitudes involve different regimes, characterized by the so-called Knudsen number $K n$. This adimensional number measures the ratio between the average time separating two successive collisions of a given particle and a characteristic time of the external flow. At altitudes of $70 \mathrm{~km}$ or below, this Knudsen number is very small, and the flows are described by the Navier-Stokes equations. It is well known that Navier-Stokes equations cease to be valid for higher altitudes corresponding to Knudsen numbers larger than $10^{-3}$. At this level, slip effects can be observed in the boundary layer and the gas gets rarefied in the wake. For example, such slip effects can be observed in Figure 1, where a kinetic calculation carried over a wedge plate at high Mach number predicts a tangential velocity at the wall of the order of $100 \mathrm{~m} / \mathrm{s}$ for a velocity at infinity equal to $1477 \mathrm{~m} / \mathrm{s}$. Such effects can lead to significant changes in the aerodynamic coefficients of the vehicle.

The standard solution is to use analytical slip boundary conditions as described in [9], $[6],[22]$. But the constants which are involved are hard to identify and their validity is questionable. On the other hand, a direct simulation of the kinetic problem is rapidly too expensive, because it requires one computational cell per mean free path. To overcome such difficulties, many authors have recently tried to use intermediate asymptotic models such as Burnett equations [23].

The solution proposed herein is quite different. It uses locally a kinetic model in the boundary layer coupled to a global Navier-Stokes solver. The coupled problem is solved by the time marching algorithm introduced and studied in [12] and [19]. The coupling can be achieved either by friction (present work) or by half fluxes [2]. A complete analysis of the coupling strategy, summarizing and developping the results obtained in the phase Research and Development of the European space program Hermès is described in [19]. In [19], the model used to solve Boltzmann equation is simply the hard sphere model and for Navier-Stokes the viscosity is assumed to be constant, while in the present work 
more sophisticated physical models on both the viscosity and internal energy are used. In Section 2, we describe the kinetic governing equation with an emphasis on the transition regime, followed in Section 3 by a brief description of the Navier-Stokes equations. In Section 4, we describe the coupling strategy. The following section describes the global Navier-Stokes solver. The numerical method used to solve the Boltzmann equation is introduced in Section 6. Numerical results are presented in Section 7. And finally, we end this paper by concluding remarks.

\section{Boltzmann equation}

Let $f$ be the density of gas particles at position $x$ with velocity $v$, and internal energy $I$. The Boltzmann equation of rarefied gas dynamics characterizes this density as the solution of the integrodifferential equation ([7])

$$
\frac{\partial f}{\partial t}+v \frac{\partial f}{\partial x}=Q(f, f)
$$

For molecular gas having internal degrees of freedom, the collision operator $Q$ is defined by

with

$$
Q(f, f)=\int_{\Delta}\left(f^{\prime} f_{*}^{\prime}\left(\frac{I I_{*}}{I^{\prime} I_{*}^{\prime}}\right)^{\delta-1}-f f_{*}\right) B d v_{*} d I_{*} \varphi_{\delta}(r) d r \psi_{\delta}(R) d R d \omega
$$

$$
\begin{gathered}
\Delta=I R^{3} \times I R_{+} \times[0,1]^{2} \times S^{2} \\
\varphi_{\delta}(r)=[r(1-r)]^{\frac{\delta}{2}-1}, \psi_{\delta}(R)=R^{2}\left(1-R^{2}\right)^{\delta-1} .
\end{gathered}
$$

As usual we have used the notation $f=f(v, I), f^{\prime}=f\left(v^{\prime}, I^{\prime}\right), f_{*}=f\left(v_{*}, I_{*}\right) \ldots$, with $\left(v_{*}, I_{*}\right)$ the velocity and internal energy of the colliding particle, and $\left(v^{\prime}, v_{*}^{\prime}\right)$ and $\left(I^{\prime}, I_{*}^{\prime}\right)$ the post collision velocities and internal energies. As in the monoatomic case, the collision direction $\omega \in S^{2}$ is fixed and in a collision, we transform the vector $\left(v, v_{*}, I, I_{*}\right)$ with $v, v_{*} \in I R^{3}, I, I_{*} \geq 0$, by setting

$$
\begin{gathered}
e^{2}=\frac{1}{4}\left|v-v_{*}\right|^{2}+I^{2}+I_{*}^{2}=\text { total energy of the collision, } \\
g=v-v_{*}=\text { relative velocity, }
\end{gathered}
$$

and by defining the post collision velocities $\left(v^{\prime}, v_{*}^{\prime}\right)$ and energies $\left(I^{\prime}, I_{*}^{\prime}\right)$ by

$$
\begin{gathered}
v^{\prime}+v_{*}^{\prime}=v+v_{*}, \\
g^{\prime}=v^{\prime}-v_{*}^{\prime}=2 \operatorname{Re}\{g-2 \omega g \cdot \omega\} /|g|, \\
I^{\prime 2}=r\left(1-R^{2}\right) e^{2}, \quad I_{*}^{\prime 2}=(1-r)\left(1-R^{2}\right) e^{2} .
\end{gathered}
$$

The factors $R . r \in[0,1]$ introduced in the collision operator determine the quantity of energy which is exchanged between internal and kinetic energy and between the two internal energies ([5]). The practical form of $\varphi_{\delta}$ and $\psi_{\delta}$ given here are such that the corresponding measure is invariant in the collision process. The term $\left(I I_{*} / I^{\prime} I_{*}^{\prime}\right)^{\delta-1}$ is introduced to give the right value of

$$
\gamma=\frac{\delta+5}{\delta+3}
$$


in the limiting hydrodynamic equation of state $p=(\gamma-1) \rho e$.

The collision cross section $B$ measures the probability of collision of particles $(v, I)$ and $\left(v_{*}, I_{*}\right)$ with parameters $(\omega, r, R)$. In the general case, it is a function of all collision invariants

$$
B:=B\left(e, R|g|, R|g \cdot \omega|, I^{2} r\left(1-R^{2}\right), I_{*}^{2}(1-r)\left(1-R^{2}\right),\left(1-R^{2}\right)\left(I^{2}+I_{*}^{2}\right)\right)>0 .
$$

In our simulations, we have used the classical Variable Hard Sphere model (VHS)

$$
B=C|g|^{-2 \alpha}|g \cdot \omega| R^{1-2 \alpha},
$$

which is the simplest model compatible with a Sutherland type viscosity law

$$
\mu=K T^{\frac{1}{2}+\alpha}
$$

at the Navier-Stokes limit.

This equation must be complemented by boundary conditions imposing the distribution of incoming particles. In the case of perfect accomodation on the body's surface, we would have

$$
\begin{gathered}
f(x, v, I, t)=\rho_{\infty} M_{u_{\infty}, T_{\infty}}(v, I) \quad \text { if } v \cdot n<0 \text { at infinity, } \\
f(x, v, I, t)=k M_{u_{w}, T_{w}}(v, I) \text { if } v \cdot n<0 \text { on the body's surface, } \\
\int f(x, v,, I, t) v \cdot n d v d I=0 \text { on the body's surface, }
\end{gathered}
$$

with $M_{u, T}$ denoting the Maxwellian distribution with mean velocity $u$ and temperature $T$

$$
M_{u, T}(v, I)=\lambda_{\delta} \frac{\rho I^{\delta-1}}{T^{(3+\delta) / 2}} e^{-\frac{|v-u|^{2}+2 I^{2}}{2 T}}
$$

More elaborate boundary conditions are introduced in Section 6 . In any case, when the gas is dense, solving Boltzmann equation is very expensive. Hence it is more convenient to solve Navier-Stokes equations. When the gas is nearly dense the use of Navier-Stokes model with an appropriate boundary conditions derived from the kinetic theory may give a good result (see the next paragraph for a brief discussion and [22], [6], [9] or [8], for more details on the derivation).

\section{Navier-Stokes equations with slip boundary conditions}

The Navier-Stokes equations are given in conservative form by

$$
\frac{\partial W}{\partial t}+\nabla \cdot F(W)=0 \quad \text { on } \quad \Omega
$$

with the state vector $W$ and flux $F(W)$ given by

$$
W=\left[\begin{array}{c}
\rho \\
\rho u \\
\rho\left(e+\frac{u^{2}}{2}\right)
\end{array}\right]
$$




$$
F(W)=\left[\begin{array}{c}
\rho u \\
\rho u \otimes u+p I d-\sigma_{v} \\
\left(\rho\left(e+\frac{u^{2}}{2}\right)+p\right) u-\sigma_{v} \cdot u+q
\end{array}\right] .
$$

Moreover, the internal energy $e$ is related to the gas density $\rho$ and pressure $p$ by the equation of state $p=(\gamma-1) \rho e$, the viscous stress $\sigma_{v}$ is proportional to the deviatoric part of the deformation rate tensor, and the heat flux $q$ is related to the temperature gradient by the Fourier law :

$$
\begin{gathered}
\sigma_{v}=\mu(T)\left(\nabla u+\nabla^{t} u\right)-\lambda(T) \operatorname{div} u I, \\
q=-\lambda_{T}(T) \nabla T .
\end{gathered}
$$

Here, $\Omega$ is the physical domain, on which we impose the boundary conditions

$$
W=W^{\infty} \text { at infinity }
$$

and appropriate boundary conditions on the body as specified below.

The standard approach in rarefied regimes is to use the following slip boundary conditions on the body :

$$
\begin{gathered}
u \cdot n=0, \\
\rho u \cdot \tau=K_{n} C_{1 \beta} \partial_{n}(u \cdot \tau)+K_{n} C_{6 \beta} \partial_{\tau} T, \\
T-T_{b o d y}=K_{n} C_{2 \beta} \partial_{n} T .
\end{gathered}
$$

Above $n$ denotes the unit normal vector to the wall and $\tau$ any tangential direction.

Such boundary conditions can be obtained as in Gupta, Moss and Scott [9] by assuming that half-flux are conserved in the kinetic boundary layer. Alternatively, for monoatomic gases, these boundary conditions are obtained in Coron [8] by solving the Boltzmann equation at order $\left(K_{n}^{2}\right)$, approximating $f$ by the asymptotic equation

$$
\begin{aligned}
F_{\varepsilon}(x, v, t) & =M_{u, T}(v)\left[\rho-K_{n} \phi(x, v, t)\right]+\chi\left(\frac{d(x, \text { body })}{K_{n}}, v, t\right) \\
& +K_{n}^{2} C(x, v, t), \\
K_{n} \phi(x, v, t) & =\frac{2}{5} \frac{\lambda}{\rho(r T)^{2}}\left(\frac{(v-u)^{2}}{2 r T}-\frac{5}{2}\right)(v-u) \cdot \operatorname{grad} T \\
& -\frac{\mu}{\rho(r T)^{2}}\left((v-u) \otimes(v-u)-\frac{1}{3}(v-u)^{2} \mathrm{Id}\right): \operatorname{grad} u .
\end{aligned}
$$

Above, $M_{u, T}(v)$ is the standard Maxwellian distribution, $\phi$ is the Chapman-Enskog correction used in the derivation of the Navier-Stokes equation [8] and $\chi$ is a boundary layer correcting term.

The boundary conditions (2) and (3) involve constants $C_{i \beta}$ which are hard to identify and their derivation uses a priori assumptions which turn out to be quite arbitrary. Moreover, their extension to diatomic gases is quite delicate. Hence we would like to replace them by flux boundary conditions of the type

$$
\begin{gathered}
\sigma_{v} \cdot \tau=g_{1}, \\
-q \cdot n+u_{\tau} \cdot \sigma_{v} \cdot n=g_{2},
\end{gathered}
$$


where the friction stress vector $g_{1}$ and energy flux $g_{2}$ would be computed numerically by a local kinetic model.

The proposed method is therefore to couple these Navier-Stokes equations to the Boltzmann equation, where Navier-Stokes equations are used in their domain of validity far away from the obstacle and the Boltzmann equation is used in a small domain surrounding the obstacle. The friction fluxes are then obtained from the Boltzmann equations and plugged as wall boundary conditions in the global Navier-Stokes system.

\section{Boltzmann/Navier-Stokes coupling}

\subsection{Coupled problem}

Let us consider the geometry described in figure 2. Let $f(x, v, I, t)$ denotes the particle distribution in the Boltzmann region $\Omega_{V}$, which is a small region surrounding the body. Let $W=\left(\rho, \rho u, \rho\left(e+\frac{u^{2}}{2}\right)\right)$ be the value of the conservative variable as computed by a Navier-Stokes model in the whole domain $\Omega$. On $\Omega_{V}$, we solve the Boltzmann equation

$$
\frac{\partial f}{\partial t}+v \frac{\partial f}{\partial x}=Q(f, f)
$$

with boundary conditions

$$
\begin{gathered}
\left.f(v)\right|_{s}=M_{u, T}(v, I) \quad \text { if } \quad v \cdot n<0, \\
\left.f(v)\right|_{\text {body }}=k M_{u_{w, T}}(v, I) \quad \text { if } \quad v \cdot n<0 .
\end{gathered}
$$

Everywhere in $\Omega$, we solve the Navier-Stokes equations

$$
\frac{\partial W}{\partial t}+\operatorname{div} F(W)=0,
$$

with flux boundary conditions

$$
\begin{gathered}
W=W^{\infty} \text { at infinity } \\
F(W) \cdot n=\left[\begin{array}{c}
0 \\
n \cdot \sigma(W) \cdot n \\
\tau \cdot \sigma_{B o l} \cdot n \\
-q_{B o l} \cdot n
\end{array}\right] \quad \text { on the body. }
\end{gathered}
$$

Here, $\tau \cdot \sigma_{B o l} \cdot n$ and $q_{B o l} \cdot n$ are the total friction fluxes predicted and computed by the Boltzmann model on $\Omega_{V}$, and $(\rho, u, T)$ on the interface $S$ are the density, velocity and temperature locally predicted by the Navier-Stokes model.

Since the Boltzmann equation behaves in space like a first order transport equation, imposing the velocity distribution of the incoming particles completely defines the Boltzmann solution inside $\Omega_{V}$. Similarly, the above boundary conditions on $W$ define a well posed Navier-Stokes problem ([19], chapter 6, and [21]). Indeed, in such a problem, we can impose either a zero normal mass flux or a normal stress, either a tangential velocity or a tangential stress, and either a given temperature or a given energy flux. The combination zero mass flux, tangential stress and heat flux is thus perfectly appropriate. 


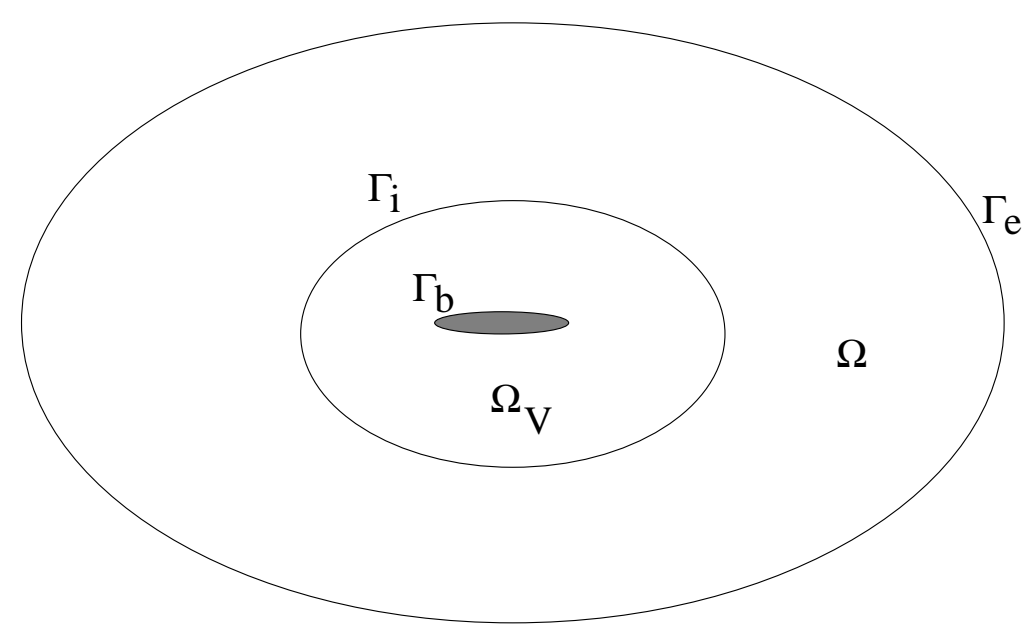

Figure 2: The global geometry

The coupling from the Boltzmann equation into the Navier-Stokes model is achieved by these imposed tangential and heat fluxes at the wall. The Navier-Stokes model acts on Boltzmann by imposing the incoming distribution $M_{u, T}(v)\left(\rho-K_{n} \phi\right)$ on the interface $S$.

The proposed coupled model is therefore well defined. With the same global NavierStokes solver, it gives an easy way of suplementing and testing a large variety of kinetic boundary conditions. These kinetic boundary conditions are first imposed on the Boltzmann model, and the resulting fluxes $\sigma_{B o l}$ and $q_{B o l}$ are then plugged in the Navier-Stokes equations. They correspond to the losses in tangential momentum and energy of particles colliding into the wall.

\subsection{Interpretation}

The interpretation of the above coupled problem is easy when coupling the same equation on overlapping domains. If we make additional assumption, similar argument, as has been seen in [19] and [20] for Navier-Stokes/Navier-Stokes coupling can still hold for the Boltzmann/Navier-Stokes coupling. For example, we can suppose that Boltzmann and Navier-Stokes equations correspond to the same physical problem inside $\Omega_{V}$. Then $W$ is solution of the same physical problem on $\Omega_{V}$ as is $f_{l o c}$, and is characterized by a set of boundary conditions (quasi Maxwell distribution on the interface, imposed friction forces on $\Gamma_{b}$ ), which are also satisfied by $f_{l o c}$. Therefore, it should correspond to the same physical solution. By construction, this physical solution then satisfies the Navier-Stokes equations on $\Omega$ (because $W$ does) the imposed inflow conditions on $\Gamma_{\infty}$ (also because of $W$ ), and adequate kinetic boundary conditions on the wall (those imposed on $f_{l o c}$ ). Compared to a standard Navier-Stokes approach and under the assumption on the equivalence between Navier-Stokes and Boltzmann, we have finally replaced the unknown wall boundary conditions on the velocity by a well defined kinetic boundary conditions imposed on $f_{l o c}$ and through $f_{l o c}$, we also have some information on the kinetic structure of the flow next to the wall. 
Even if we do not believe in the equivalence between Navier-Stokes and Boltzmann models inside $\Omega_{V}$, we can still justify our coupling strategy provided that we assume that imposing friction forces on $\Gamma_{b}$, given inflow data on $\Gamma_{\infty}$ and using either Boltzmann equations or Navier-Stokes equations on $\Omega$ lead to the same kinetic physical solution $f_{\text {glo }}$ outside $\Omega_{V}$. (For the Boltzmann model, we would supplement the friction forces by an additional information extracted from $f_{l o c}$ in order to get a well posed global problem). Then $f_{l o c}$ and $f_{g l o}$ would satisfy :

equality of velocity distribution on interface, equality of friction forces (+ additional information) on the wall, the same Boltzmann equation on $\Omega_{V}$.

We would deduce as for the Navier-Stokes/Navier-Stokes coupling ([19] and [20]) that the two distributions $f_{l o c}$ and $f_{g l o}$ are equal inside the local domain and in particular at the wall. Then, $f_{g l o}$ satisfying the kinetic conditions imposed to $f_{l o c}$ on the wall, the Boltzmann equation on $\Omega$ and the adequate inflow boundary conditions on $\Gamma_{\infty}$, is the desired Boltzmann solution. In turn, this means that $f_{l o c}$ is locally equal to the desired solution. Therefore, if our assumption is true, $f_{l o c}$ is locally the kinetic solution which has been computed at low cost by using a coarse averaged approximation away from the wall (outside $\Omega_{V}$ ).

\subsection{Algorithm}

The numerical solution of this coupled model can be easily achieved by the following algorithm :

Initialization

0) Solve Navier-Stokes equations on the whole domain (using a coarse mesh, a conservative formulation, flux splitting and say slip boundary conditions).

Loop on time : For increasing time $n$ and until reaching a steady state,

1. Solve several time steps of the local Boltzmann solver

$$
\begin{gathered}
\left(f_{n+1}-f_{n}\right) / D T+v \cdot \nabla f_{n}=Q\left(f_{n}, f_{n}\right), \\
f_{n+1}^{-}=M_{u, T}(v)\left(\rho-K_{n} \phi\right) \text { on the interface } S, \\
f_{n+1}^{-}=k M_{u_{w, T}}(v) \text { on the body. }
\end{gathered}
$$

2. From $f_{n+1}$ compute the friction fluxes $F_{b}=\left(\tau \cdot \sigma_{B o l} \cdot n,-q_{B o l} \cdot n\right)$ on the body.

3. With imposed friction fluxes $F_{b}$, solve several time steps of the global Navier-Stokes equations

$$
\begin{gathered}
\left(W_{n+1}^{g}-W_{n}^{g}\right) / D T+F_{i, i}\left(W_{n+1}^{g}\right)=0, \\
F\left(W_{n+1}^{g}\right) \cdot n=\left(0, n \cdot \sigma\left(W_{n+1}^{g}\right) \cdot n, F_{b}\right) \text { on the internal boundary } \Gamma_{b}
\end{gathered}
$$

and with the usual boundary conditions at infinity. 
The whole strategy has been proposed in the phase Research and Development of the Hermes program (see [19]). At that time it was tested with a frozen viscosity in the NavierStokes domain and with a monoatomic hard sphere model in the Boltzmann region. This still will be the case when comparing Navier-Stokes with slip kinetic boundary conditions to a Boltzmann calculation. However more sophisticated models are also studied here. This includes variable viscosity for the Navier-Stokes solver, the variable hard sphere model and the Larsen-Borgnakke model for the Boltzmann solver ([3]). These enhanced models are then validated on several configurations as will be seen in the next sections.

\section{Navier-Stokes solver}

Let us consider the compressible Navier-Stokes equations which we formally write as

$$
\frac{\partial W}{\partial t}+\operatorname{div}[F(W)]=0 \quad \text { on } \quad \Omega,
$$

with $W=\left(\rho, \rho v, \rho\left(e+\frac{u^{2}}{2}\right)\right)$ the conservative variables, and $F=F^{c}+F^{d}$ the total flux (convective and viscous part). The problem consists in computing a steady solution of these equations, satisfying the boundary conditions introduced in the previous section

$$
\begin{gathered}
W=W^{\infty} \text { at infinity, } \\
F(W) \cdot n=\left[\begin{array}{l}
0 \\
n \cdot \sigma(W) \cdot n \\
\tau \cdot \sigma_{B o l} \cdot n \\
-q_{B o l} \cdot n
\end{array}\right] \quad \text { on the body. }
\end{gathered}
$$

The global domain $\Omega$ is discretized using node centered cells (figure 3) defined on an unstructured grid. Then, at each time step $n$ and for each cell $i$, we solve

$$
\begin{aligned}
& \int_{C_{i}} \frac{W^{n+1}-W^{n}}{\Delta t}+\sum_{j \in V(i)} \int_{\partial C_{i} \cap \partial C_{j}} F^{c}\left(W^{n+1}\right) \cdot n_{i} \\
& +\int_{\partial C_{i}-\Gamma} F^{d}\left(W^{n+1}\right) \cdot n_{i}+\int_{\partial C_{i} \cap \Gamma_{\infty}} F\left(W^{n+1}\right) \cdot n_{i}=-\int_{\partial C_{i} \cap \Gamma_{b}} F \cdot n_{i} .
\end{aligned}
$$

In our numerical implementation, the fluxes $F^{c}$ and $F^{d}$ are computed at time step $n+1$ and linearized, with $F^{c}$ computed by an Osher approximate Riemann solver [16]. The resulting linear system is solved by a block relaxation method. by

On the body $\Gamma_{b}$, because of our special choice of boundary conditions, the flux is given

$$
\int_{\partial C_{i} \cap \Gamma_{b}} F \cdot n_{i}=\int_{\partial C_{i} \cap \Gamma_{b}}\left(\begin{array}{c}
0 \\
n_{i} \cdot \sigma\left(W^{n+1}\right) \cdot n_{i} \\
n_{i} \cdot \sigma_{B o l} \cdot \tau_{i} \\
-q_{B o l} \cdot n_{i}
\end{array}\right) .
$$

In other words, friction forces and heat flux are given explicitly as predicted by the local solver and the mass flux is imposed to zero. Then, in order to have a well-posed problem, 


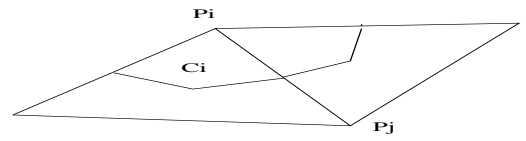

Figure 3: A boundary cell

the normal stress (the multiplier of the zero mass flux constraint) cannot be imposed and must be obtained from the solution $W^{n+1}$ (see [19] and [21]).

Remark : Imposing friction forces to the global solution instead of no slip boundary conditions allows to have an accurate solution away from the boundary layer even with a coarse mesh (see [19]).

Remark : In some of our tests, we will use a SUPG finite element method for solving (4), as introduced in [15].

\section{Boltzmann solver}

In this section, we will present first the numerical approximation methods used to solve the Boltzmann equation. Then we describe the treatment of the boundary conditions and the calculation of momentum and energy fluxes on the body.

\subsection{Approximation of the Boltzmann equation}

We have chosen to solve the Boltzmann equation by the particle method proposed by Babovski [1] and described in [4]. The main ingredient is to decouple at each time step the transport phase from the collision phase.

The free transport phase solves

$$
\frac{\partial f}{\partial t}+v \nabla_{x} f=0, \quad f\left(t_{n}\right)=f_{n},
$$

the collision phase solves

$$
\frac{\partial f}{\partial t}-Q(f, f)=0, \quad f\left(t_{n}\right)=f_{n+1}^{\text {transport }}
$$

These two steps are realized successively. For this purpose, particle approximations of kinetic equations are introduced, based on the approximation of the density $f$ by a sum 
of Dirac mass

$$
f(t, x, v, I)=\frac{1}{n_{\infty}} \sum_{i=1}^{N} \delta\left(x-x_{i}(t)\right) \delta\left((v, I)-\left(v_{i}, I_{i}\right)(t)\right) .
$$

The positions $x_{i}(t)$ change during the free transport phase only, and are updated following (6) by

$$
x_{i}(t+\Delta t)=x_{i}(t)+\Delta t v_{i}(t) .
$$

The velocities and internal energies $\left(v_{i}(t), I_{i}(t)\right)$ change during the collision step $(7)$. At this step, one covers the computational domain with regular cells $C_{i}$, and one solves the collision step separately on each cell, taking as initial distribution

$$
f(v, t=0)_{\mid C_{j}}=\frac{1}{N} \sum_{i \in C_{j}} \delta\left((v, I)-\left(v_{i}, I_{i}\right)(0)\right) .
$$

Following [1], we solve (7) under the weak form

$$
\begin{aligned}
\frac{d}{d t}<f(t), g> & =\langle Q(f, f), g> \\
& =\frac{1}{2} \int f f_{*}\left(g^{\prime}+g_{*}^{\prime}\right) B d m-\frac{1}{2} \int f f_{*}\left(g+g_{*}\right) B d m
\end{aligned}
$$

where $g$ is any continuous function and where we have used the notation

$$
\begin{aligned}
<f, g> & =\int_{I R^{3} \times I R_{+}} f(v, I) g(v, I) d v d I, \\
d m & =d v d v_{*} d I d I_{*} \varphi_{\delta} \psi_{\delta} d r d R d \omega .
\end{aligned}
$$

The above writing of the collision operator is a consequence of the microreversibility of each individual collision and stays valid even if $f$ is a sum of Dirac distributions. For our choice of $f$, the scattering term is then simply

$$
\frac{1}{2} \int f f_{*}\left(g+g_{*}\right) B d m=\frac{\rho^{2}}{8 N^{2}} \sum_{i, j}\left[g\left(v_{i}, I_{i}\right)+g\left(v_{j}, I_{j}\right)\right] W_{v_{i}, v_{j}},
$$

with weight $W_{v_{i}, v_{j}}$ given by

$$
W_{v_{i}, v_{j}}=\int B \varphi_{\delta} \psi_{\delta} d r d R d \omega
$$

The source term in (9) is similarly

$$
\frac{1}{2} \int f f_{*}\left(g^{\prime}+g_{*}^{\prime}\right) B d m=\frac{\rho^{2}}{8 N^{2}} \sum_{i, j} \int\left[g\left(v_{i}^{\prime}, I_{i}^{\prime}\right)+g\left(v_{j}^{\prime}, I_{j}^{\prime}\right)\right] B \varphi_{\delta} \psi_{\delta} d r d R d \omega,
$$

with $v_{i}^{\prime}, v_{j}^{\prime}, I_{i}^{\prime}, I_{j}^{\prime}$ the result of the collision of $\left(v_{i}, I_{i}\right)$ with $\left(v_{j}, I_{j}\right)$.

We then integrate (7) in time by :

- replacing the double sum in (10) or (11) by a random choice of $N / 2$ pairs of particles

$$
\sigma=\left\{\left(v_{1}, I_{1}\right),\left(v_{* 1}, I_{* 1}\right)\right\},\left\{\left(v_{2}, I_{2}\right),\left(v_{* 2}, I_{* 2}\right)\right\}, \ldots\left\{\left(v_{N / 2}, I_{N / 2}\right),\left(v_{* N / 2}, I_{* N / 2}\right)\right\} ;
$$


- discretizing (7) in time by the explicit Euler scheme

$$
\begin{aligned}
<f^{n+1}, g>= & <f^{n}, g>+\Delta t[\text { source- scattering }] \\
= & \frac{1}{N} \sum_{i=1}^{N / 2}\left[g\left(v_{i}, I_{i}\right)+g\left(v_{* i}, I_{* i}\right)\right]\left(1-\frac{N-1}{N} \Delta t W_{v_{i}, v_{*} i}\right) \\
& +\frac{N-1}{N} \Delta t \int\left[\varphi\left(v_{i}^{\prime}, I_{i}^{\prime}\right)+\varphi\left(v_{* i}^{\prime}, I_{* i}^{\prime}\right)\right] B_{i} \varphi_{\delta} \psi_{\delta} d r d R d \omega ;
\end{aligned}
$$

- using the identities ( assume that $\varphi_{\delta}(r) \psi_{\delta}(R) d r d R d \omega$ is normalized so as to be a probability measure)

$$
\begin{gathered}
\frac{N-1}{N} \Delta t B_{i}=\int 1_{0 \leq s \leq(N-1) / N B_{i} \Delta t} d s \\
1-\frac{N-1}{N} \Delta t W_{v_{i}, v_{* i}}=\iint 1_{(N-1) / N B_{i} \Delta t \leq s \leq 1} d s \varphi_{\delta} \psi_{\delta} d r d R d \omega,
\end{gathered}
$$

- and computing all integrals using a Monte-Carlo method.

In practice, once the random choice of the $N / 2$ pairs has been made, we perform for each pair a random choice of $r, R, \omega$ according to the law $\varphi_{\delta} \psi_{\delta} d r d R d \omega$, and a uniformly random choice of $s_{i} \in(0,1)$. Then, if $s_{i} \leq \frac{N-1}{N} \Delta t B_{i}$, the source term $1-\frac{N-1}{N} \Delta t W_{v_{i}, v_{* i}}$ is zero, the scattering term $\frac{N-1}{N} \Delta t B_{i}$ is equal to one, and thus the collision is processed : the particles $(v, I)_{i},\left(v_{*}, I_{*}\right)_{i}$ are replaced by the particles $\left(v^{\prime}, I^{\prime}\right)_{i},\left(v_{*}^{\prime}, I_{*}^{\prime}\right)_{i}$ obtained in the collision with parameters $r, R, \omega$. If not, the pair of particles is kept unchanged. The final distribution is then

$$
\begin{aligned}
f^{n+1}=\frac{1}{N_{o}} \quad & \sum_{i \in I_{1}}\left\{\delta\left((v, I)-\left(v_{i}, I_{i}\right)\right)+\delta\left((v, I)-\left(v_{* i}, I_{* i}\right)\right)\right\} \\
& \sum_{i \notin I_{1}}\left\{\delta\left((v, I)-\left(v_{i}^{\prime}, I_{i}^{\prime}\right)\right)+\delta\left((v, I)-\left(v_{* i}^{\prime}, I_{* i}^{\prime}\right)\right)\right\}
\end{aligned}
$$

where $I_{1}$ denotes the set

$$
I_{1}=\left\{J i, 1 \leq i \leq N / 2, s_{i}>\frac{N-1}{N} \Delta t B_{i}\right\} .
$$

The complexity of this algorithm is in $0(N)$. Moreover, by construction, this algorithm conserves exactly mass, momentum and energy.

\subsection{Numerical algorithm}

The numerical algorithm corresponds to the Monte-Carlo method described above and was initially developed at the University of Kaiserslautern. This algorithm is the following :

1. Get an initial distribution of particles.

2. Loop in time from 1 to $N_{2}$ : 
- generate randomly the particles at the external boundary ;

- advance the particles in free transport

$$
x_{i}(t+\Delta t)=x_{i}+v_{i} \Delta t
$$

- erase the particles that leave the domain, except those which get in contact with the obstacle and which are reflected as indicated later ;

- accomplish the intermolecular collision as indicated above.

As output, the average values $\rho, u, T$ and the fluxes at the body are obtained by averaging the corresponding kinetic quantities over all particles of the cell considered and on several ( $\geq 100$ ) consecutive time steps. The fluxes at the body (friction forces and heat fluxes) are calculated using their kinetic definitions. Under the notation

$n$ : external unit normal to the physical domain,

$\Gamma$ : surface section on which we integrate the flux,

$d S:$ area of $\Gamma$,

$d t$ : time interval considered,

the quantity $\sigma \cdot n d S d t$ corresponds to the impulse exerted on the fluid by the surface section $\Gamma$ during the time interval $d t$, i.e. to the sum of impulses received by the elementary particles which collide with $\Gamma$ during the time interval $d t$. We then have

$$
\sigma_{B o l} \cdot n d S d t=\sum_{i \in J} m_{i}\left(v_{i}^{+}-v_{i}^{-}\right),
$$

with $J$ denoting the set of particles which collide with $\Gamma$ during the time interval $d t$. Therefore we have the final formula:

$$
\sigma_{B o l} \cdot n=\frac{\rho^{\infty} \operatorname{vol}^{\infty}}{n^{\infty} d S d t} \sum_{i \in J}\left(v_{i}^{+}-v_{i}^{-}\right)
$$

with $\rho^{\infty}, v^{\infty} l^{\infty}$ and $n^{\infty}$ denoting respectively the density, volume, and number of particles associated with a reference cell of the flow at infinity. Similarly $q_{B o l} \cdot n d S d t=\left(n \cdot \sigma_{v} J\right.$. $u-q \cdot n) d S d t$ is the energy received by the fluid on the section $\Gamma$ during the time interval $d t$, which gives

$$
q_{B o l} \cdot n d S d t=-\sum_{i \in J}\left(\frac{1}{2} m_{i}\left|v_{i}^{+}\right|^{2}+I_{i}^{+}-\frac{1}{2} m_{i}\left|v_{i}^{-}\right|^{2}-I_{i}^{-}\right) .
$$

Remark 6.1 In order to minimize the memory place, at each call to the local Boltzmann solver, we initialize the initial distribution of the particles by the Maxwellians $M_{i}$ defined at each cell $i$ and whose parameters $\rho_{i}, u_{i}$ and $T_{i}$ are either the average values $\rho_{i}^{N-1}, u_{i}^{N-1}$ and $T_{i}^{N-1}$ computed at the previous call to the Boltzmann solver, or the values given by the present Navier-Stokes solution.. 
$\Gamma_{\mathrm{i}}$

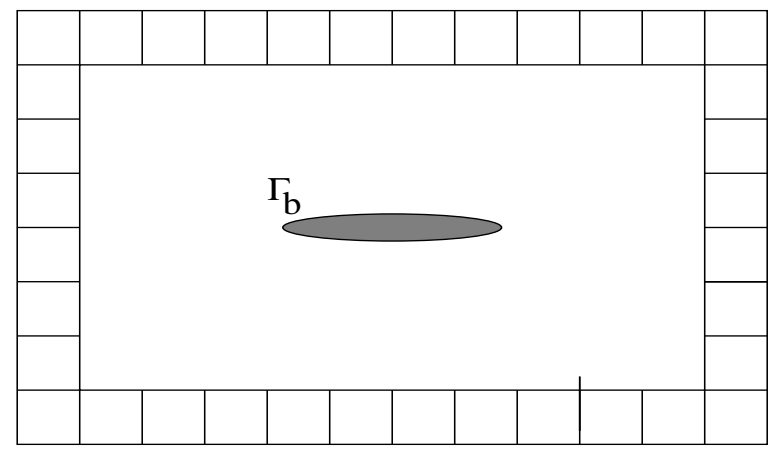

Figure 4: Layer of boundary cells

\subsection{Boundary conditions at infinity}

In classical situations, the boundary conditions at infinity consist in imposing the distribution of the ingoing particles equal to the Maxwellian at infinity. We mean by infinity that the limit of the domain is fixed far away from the obstacle.

Herein, we want to solve a local Boltzmann problem in a small domain surrounding the obstacle. In this domain, the external boundary conditions are computed from the global Navier-Stokes solver. To adapt these new boundary conditions (instead of the classical boundary conditions at infinity) to these particular geometries, we introduce a layer of boundary cells (see figure 4). We compute at the center of each cell the quantities $\rho, u$ and $T$ predicted by the current Navier-Stokes calculation. To do so, we look for the triangle in the global mesh which contains the center $i$ of the boundary cell and we compute $\rho_{i}, u_{i}$ and $T_{i}$ by linear interpolation. Then, we introduce in this cell $\left(\rho_{i} N^{\infty}\right)$ particles. These particles are distributed randomly in space, and their velocities and energies are obtained by drawing them randomly according to the Maxwellian distribution $M\left(u_{i}, T_{i}\right)$.

These particles are finally transported by their velocity field and we take into account only the particles which enter effectively the local domain during the time step considered. This operation is repeated at each time step of the local Boltzmann solver.

To be perfectly accurate, we should replace the Maxwellian distribution $M\left(u_{i}, T_{i}\right)$ by an approximation of the Chapman Enskog expansion $M\left(u_{i}, T_{i}\right)-K_{n} \phi$, but this seems to have very little effect on the global solution.

\subsection{Boundary conditions on the body}

In kinetic theory, the interaction between the gas and the body is expressed by a boundary condition acting on the density distribution $f(x, v, t)$. These interactions on the body are very complex and very hard to model. Therefore we restrict ourselves to the total accomodation model of Section 2. In certain specific tests, we have also used the intermediate Maxwell accomodation model given by

$$
\begin{gathered}
f(x, \xi, I)=\beta(x) f(x, R \xi, I)+(1-\beta(x)) \alpha(x) M_{0, T}(\xi, I) \\
\forall \xi \text { such that } \xi \cdot n>0,
\end{gathered}
$$




$$
\forall x \in \partial \Omega, \int \xi \cdot n(x) f(x, \xi, I) d \xi d I=0 .
$$

Above, $n(x)$ is the external normal to the obstacle at $\xi \in \partial \Omega, \beta(x) \in(0,1)$ is the Maxwell accomodation coefficient and

$$
R \xi=\xi-2(\xi \cdot n(x)) n(x)
$$

is the velocity of the particle after elastic reflexion on the obstacle.

This boundary condition is imposed numerically to all particles which collide into the obstacle during their free transport. They are then reflected after impact with velocity $v^{+}$ given by

$$
\begin{gathered}
v^{+}=(1-\beta) v_{a}^{+}+\beta v_{s}^{+}, \\
I^{+}=(1-\beta) I_{a}^{+}+\beta I,
\end{gathered}
$$

where $\beta$ is the accomodation coefficient, $v_{s}^{+}=R v$ is the velocity after specular reflexion and $\left(v_{a}^{+}, I_{a}^{+}\right)$is the velocity obtained assuming total accomodation, calculated by randomly drawing $(v, I)$ following the probability distribution $\lambda M_{0, T}(\xi, I)$.

\section{Numerical results}

\subsection{Sensitivity to the choice of the local domain and to the downstream boundary conditions on the global calculation}

This test case corresponds to a flow above a flat plate at no incidence with :

$$
\begin{array}{lccc}
M_{\infty} & R e_{\infty} / m & T_{\infty} & T_{\text {wall }} \\
10 . & 143.800 & 52^{\circ} \mathrm{K} & 290^{\circ} \mathrm{K}
\end{array}
$$

We compare in this paragraph the results obtained when considering a local Boltzmann domain that is smaller and smaller. More precisely, the plate takes respectively the length of $11 \mathrm{~cm}$ (L1), $10 \mathrm{~cm}$ (L2), $7 \mathrm{~cm}$ (L3) and $5 \mathrm{~cm}$ (L4) (see figure 5). The local length in the vertical projection is $1 \mathrm{~cm}$ and does not change for all configurations described above.

For all these calculations, the size of the cells in $x$ and $y$ direction is almost the same for the local calculation. The sensitivity to the choice of the local domain is studied on the friction coefficient $C_{f}$, the heat flux coefficient $C_{A}$, the slip velocities, and the normal stress vector. The viscosity law considered here is the VHS law $\mu=C T^{w}$ with $w=0.93$ and $C=6.910^{-6}$ SI.

We present on figure 6 the $C_{f}$ corresponding to the calculation $L 1, L 2, L 3$ and $L 4$. We observe that the curves obtained are perfectly identical. The heat flux corresponding to the calculation $L 1, L 2, L 3$ and $L 4$ are presented in figure 7 . The results obtained are also perfectly identical.

In conclusion, the choice of the local domain corresponding to the calculation $L 4$ is sufficient. The boundary conditions imposed on the downstream part of the plate in the global domain are satisfactory, and do not affect the solution upstream.

Remark 7.1 The number of cells in the Boltzmann calculation is 3350, the number of the Boltzmann particles is 83750, the total number of time steps for Boltzmann is 1300 , the total number of time steps for Navier-Stokes is 608, the number of coupling algorithm iterations is 5 . 


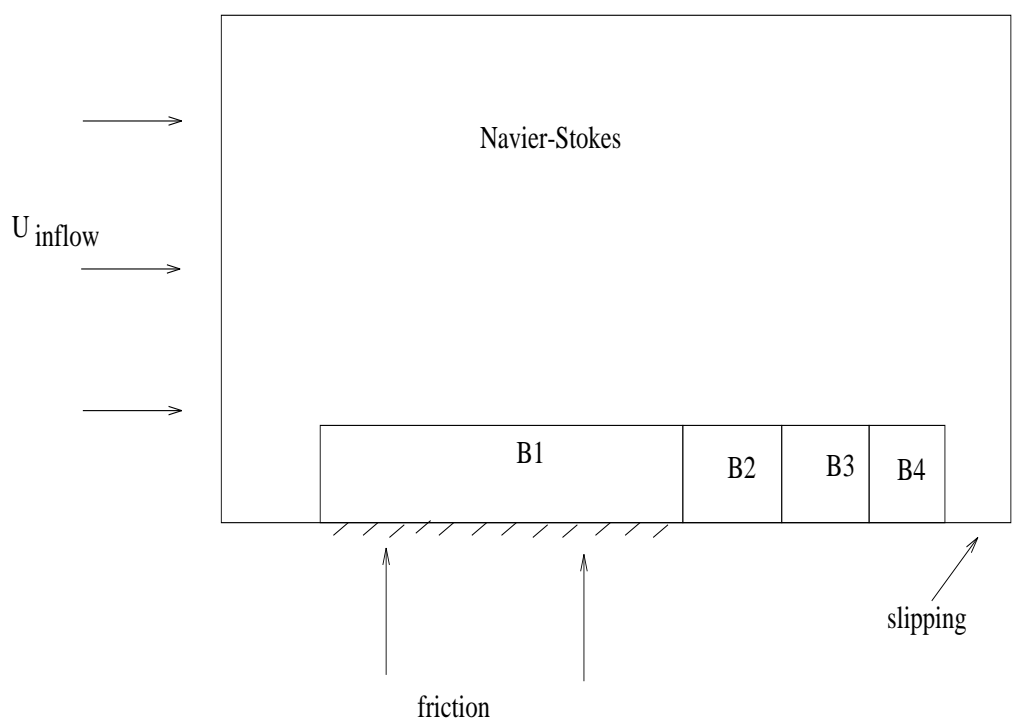

Figure 5: Coupling geometry with different local subdomains

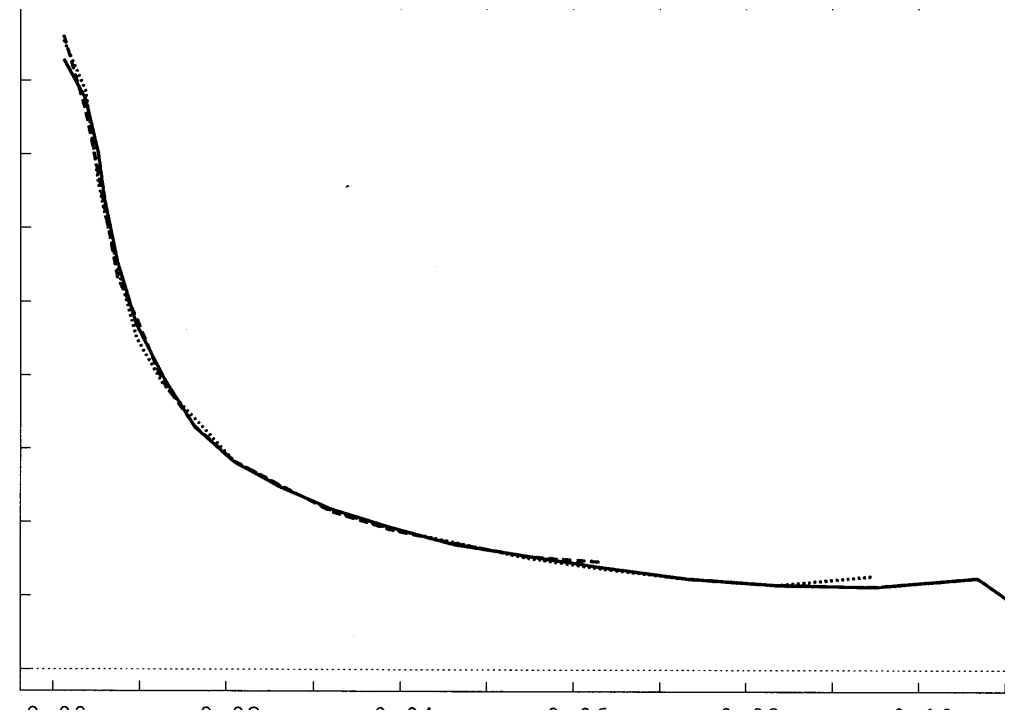

Figure 6: Values of $C_{f}$ for different subdomain sizes. Values range from 0.08 to 0.02 and all curves are perfectly superposed. 


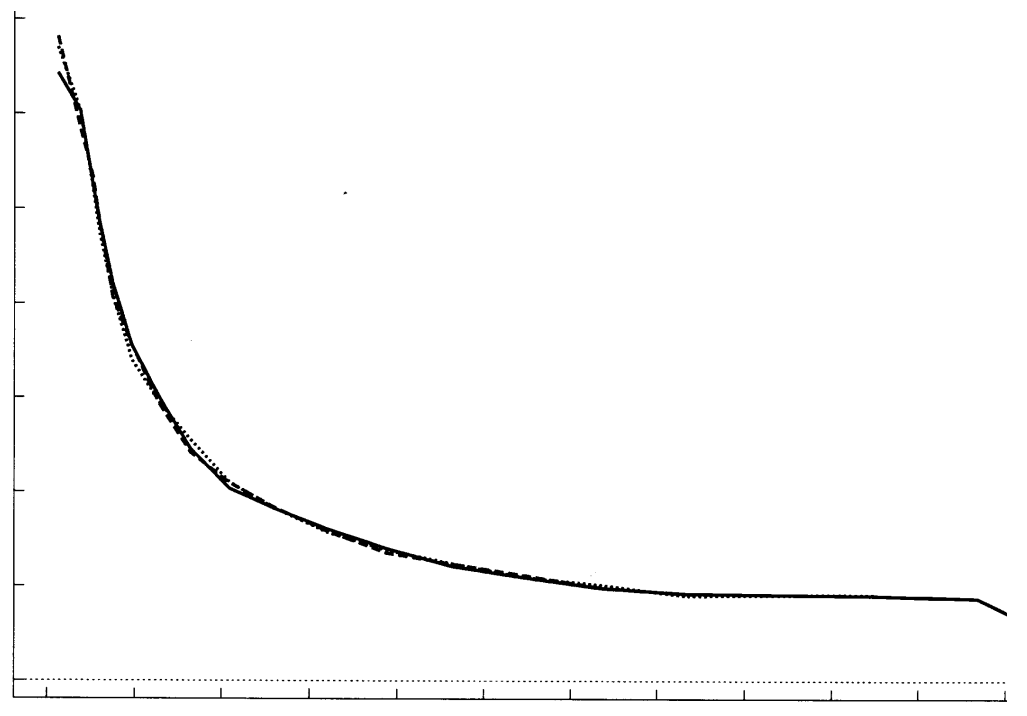

Figure 7: Values of $C_{h}$ for different subdomain sizes. Values range from 0.035 to 0.005 and all curves are perfectly superposed.

\subsection{Study of ONERA test case without angle of attack}

In this paragraph, we will compare our results to those obtained by the ONERA [10] on a $10 \mathrm{~cm}$ long flat plate at high Mach number and with no angle of attack.

The case studied corrresponds to the geometry given on figure 8 and to the following data :

Mach number $=20$,

Temperature at infinity $T_{\infty}(K)=13.6$,

Temperature at the body $T_{W}(K)=286$,

Vitesse $V_{\infty}(m / s)=1477$,

Mean free path $\lambda_{\infty}(m)=2.3410^{-4}$,

Reynolds number per meter $=83800$,

Dimensions of the Boltzmann domain $L(m)=0.081, H(m)=0.022$,

Exponent of the viscosity law $w=0.964$,

The constant of the viscosity law $C=2.1110^{-5}$.

This case has been studied by using our coupling strategy with two different NavierStokes flow solvers, and is compared to the results of a full kinetic simulation and to experimental results.

The first Navier-Stokes solver corresponds to the non-structured implicit finite volume solver described in Section 5 and based on the Osher flux splitting. The mesh of 4048 vertices is given on figure 9 . The Boltzmann solver starts from the tip of the plate and uses 8800 cells ( 250000 particles), which are of size $1 \times 0.2 \mathrm{~mm}$. The accomodation coefficient is equal to 1 at the body. We have performed 7 coupling iterations, corresponding to a total number of 2300 time steps for Navier-Stokes solver (with CFL $=20$ ) and 1800 time steps for Boltzmann solver. The total time of computation was $50 \mathrm{~h}$ on Apollo DN10000.

The second Navier-Stokes solver is a SUPG solver developped by Dassault Aviation. The mesh used for the Navier-Stokes calculation is obtained from the previous one using 


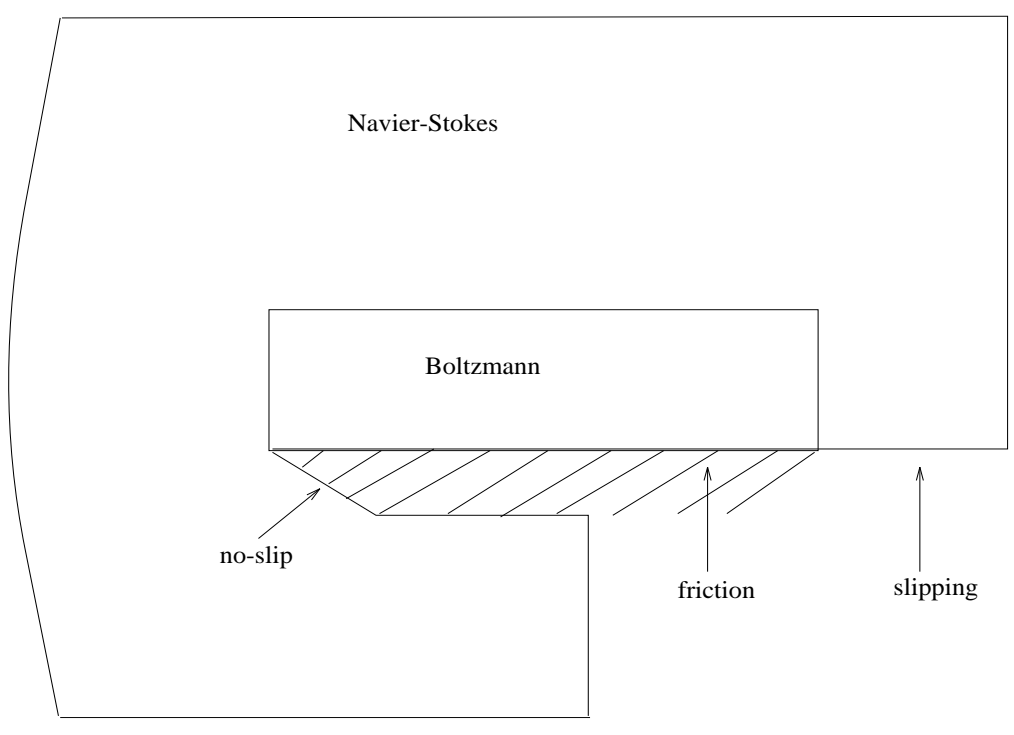

Figure 8: Plate Problem.

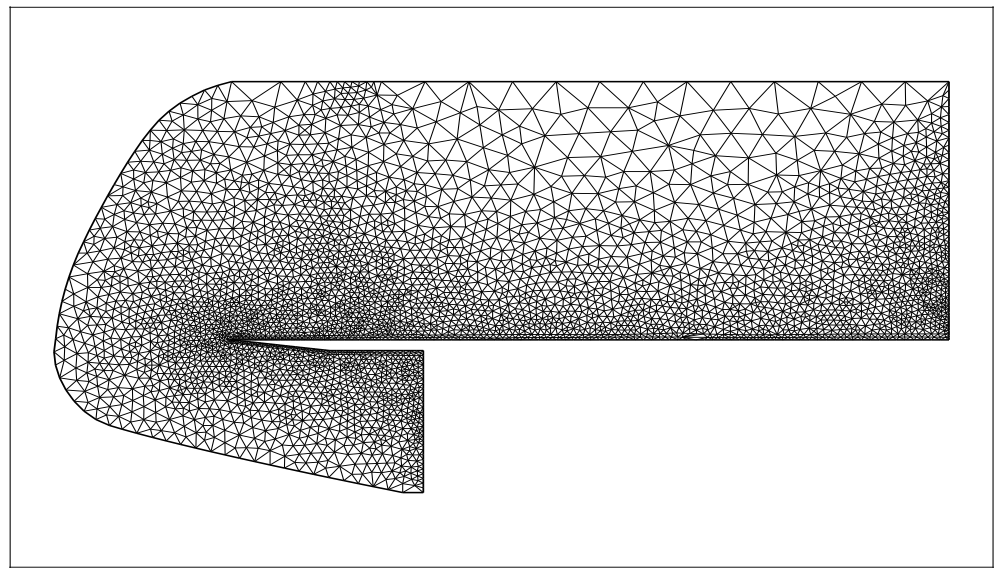

Figure 9: First mesh of the plate problem. 


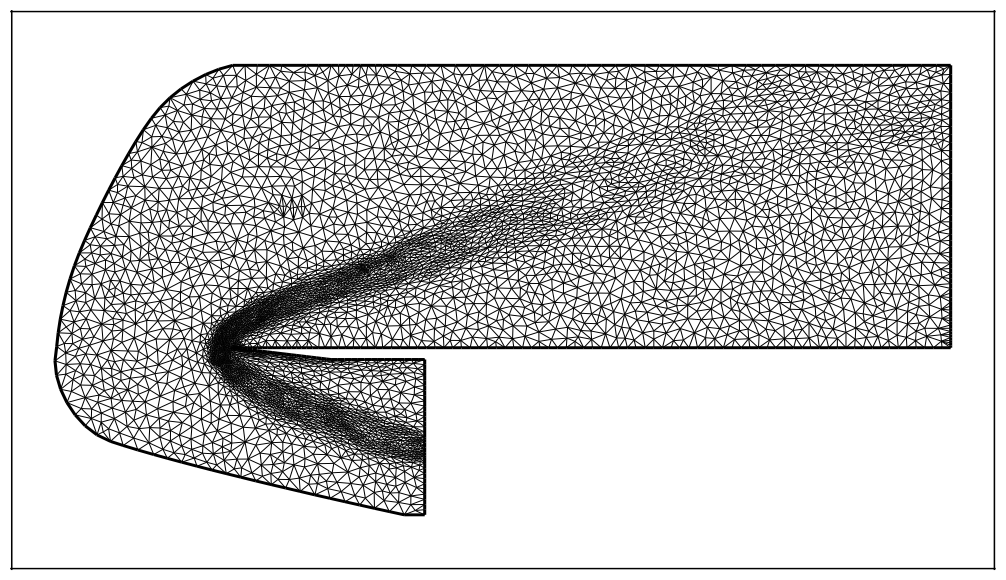

Figure 10: Second mesh of the plate problem.

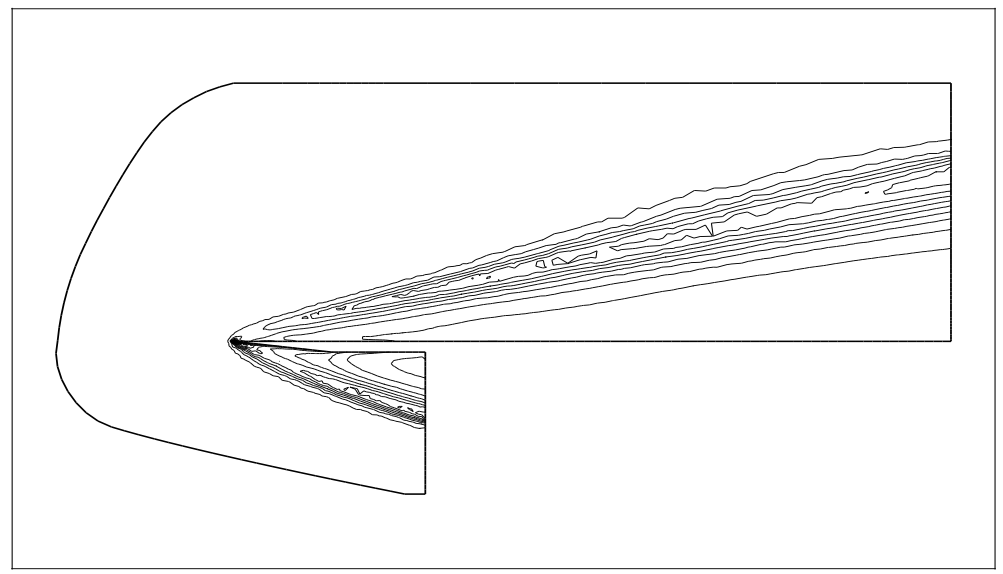

Figure 11: Isodensities for the Onera plate problem.

adaptive techniques (refined in the zone where the Hessian of the temperature is higher) as described on the figure 10. The total time of computation was $40 \mathrm{~h}$ on Apollo DN10000. The total number of coupling iterations is 5 and corresponds to 1000 time steps for the Navier-Stokes solver and 4000 time steps for the Boltzmann solver (250000 particles).

A first analysis of the results obtained by the above strategies is done by analysing the iso-densities 11, which appear to compare quite well with the available experimental data.

To refine these first observations we present the experimental density profiles and those computed by the local Boltzmann solver in the coupling strategies and in the global Boltzmann solver. These profiles are drawn at $x=2.5 \mathrm{~cm}$ on Figure 12 , and $x=7.5 \mathrm{~cm}$ on Figure 13. The solution computed in (a) (Navier-Stokes/Boltzmann, mesh 1 and accomodation equal 1) corresponds to the experimental solution except for the shock : the shock 


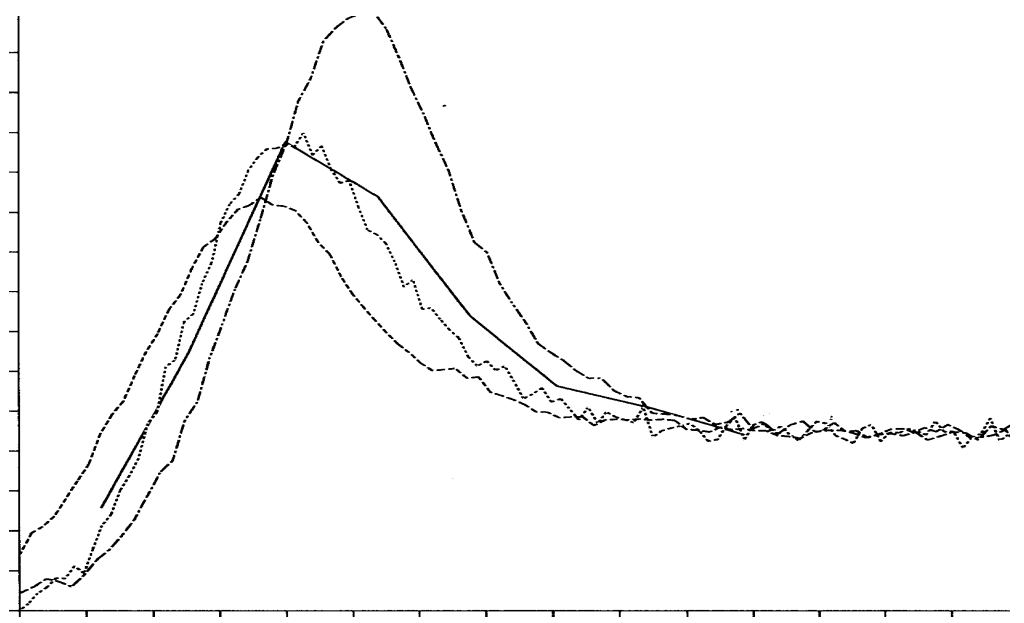

Figure 12: Density profiles at $x=0.25$. Values range from 0.70 to 1.71 . The highest curve corresponds to the coupled solution on the second mesh, the lowest curve to a full Boltzmann simulation, the intermediate curve to the coupled solution on the first mesh, and the continuous line to the experimental results.

computed at $7.5 \mathrm{~cm}$ is $2 \mathrm{~mm}$ more closer to the body than the one predicted by the experimental results. Using the adaptive mesh techniques we obtain almost identical results , but the maxima computed are slightly larger. Observe that the profiles of the numerical density and of the experimental density do not perfectly agree. Depending on the mesh we used for the Navier-Stokes equations, we arrive to obtain the same maxima levels for both approaches (the error on the shock position is then 10 to $15 \%$ ) or the same shock position (and in this case the error on the maxima levels is 10 to $15 \%$ ). Nevertheless, the agreement obtained by our strategy with the experimental results is much better than the one obtained in previous simulations based on analytic slip boundary conditions [10], and we recover the right values of velocity and temperature jumps.

\subsection{Flat Plate with an angle of attack}

The last experimental result compares our coupled strategy to a full kinetic simulation in the case of a two-dimensional flow past a $5 \mathrm{~cm}$ flat plate at a 10 degrees angle of attack. The case studied corrresponds to the following data:

Mach number at infinity $=18.31$,

Temperature at infinity $T_{\infty}(K)=13$,

Temperature at the body $T_{W}(K)=286$,

Vitesse $V_{\infty}(\mathrm{m} / \mathrm{s})=1477$.

We compare here the results of the coupled approach with those of a full kinetic simulation and to a Navier-Stokes calculation with linear slip boundary conditions, for a monoatomic gas with a hard sphere collision model. The Reynolds number is successively taken as $R e / m=60362$ corresponding to a rather dense Knudsen number of $K n=0.01$ and $R e / m=7545$ (rarefied Knudsen number $K n=0.08$ ).

The full kinetic simulation in the dense case (resp. rarefied case) was performed on a rectangular domain of $0.06 \times 0.018 m$ (resp. $0.06 \times 0.034 m$ ) discretized in 20764 (resp. 


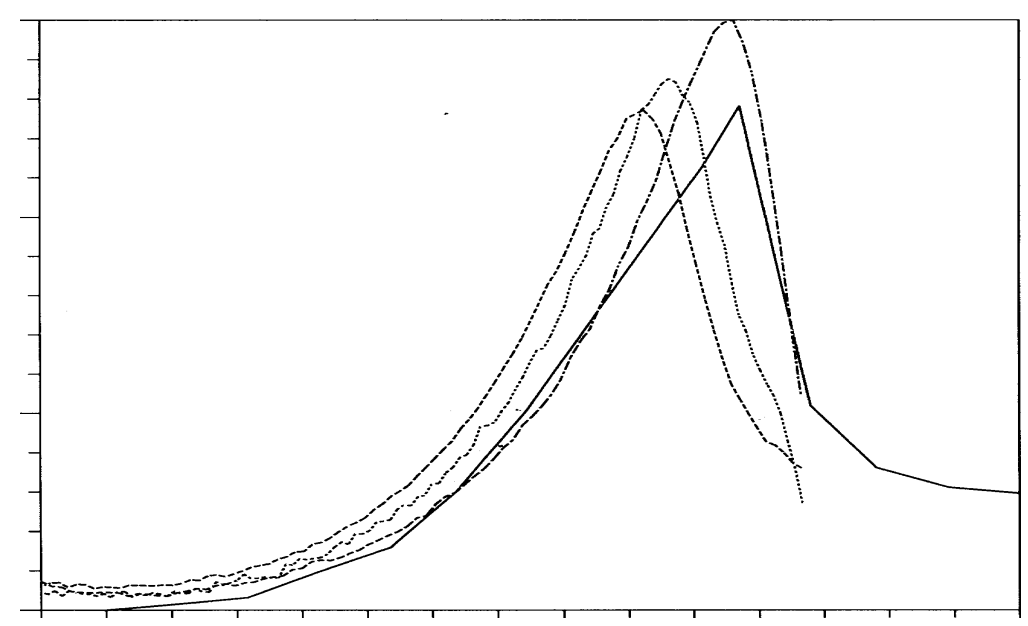

Figure 13: Density profiles at $x=0.75$. Values range from 0.55 to 2.8 . The highest curve corresponds to the coupled solution on the second mesh, the lowest curve to a full Boltzmann simulation, the intermediate curve to the coupled solution on the first mesh, and the continuous line to the experimental results.

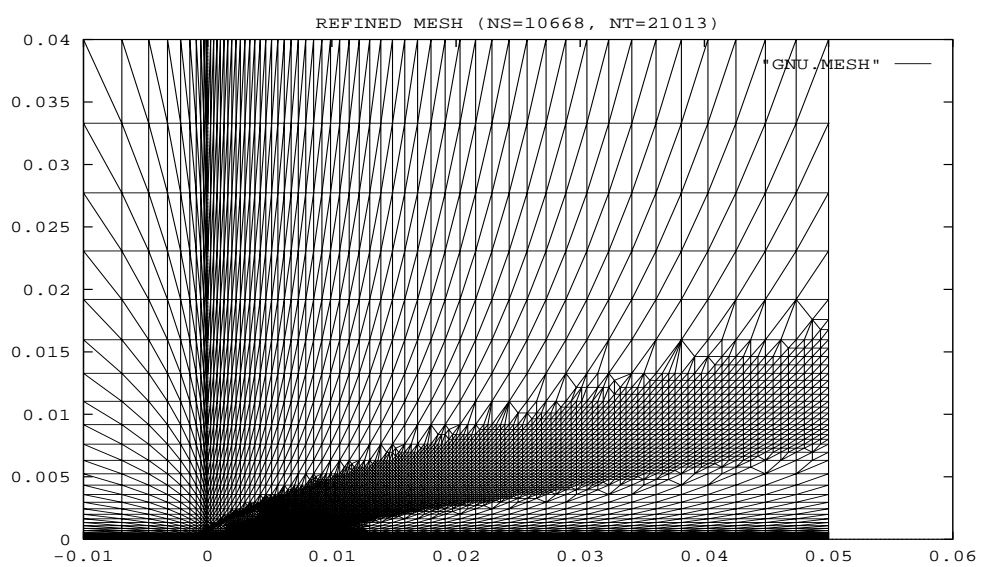

Figure 14: Mesh used for the Navier-Stokes solution over the flat plate in the dense coupled case.

10200 ) rectangular cells, using approximatively 660000 (resp. 315000 ) particles, 7000 (resp. 4500) total time steps including 6000 (resp. 4000 ) time steps for the accurate calculation of flow averages. The corresponding CPU time was of more than $20 h$ (resp. $5 h 30)$ on a $H P 735$ workstation.

The global Navier-Stokes solution with slip boundary conditions was calculated on an adaptively refined mesh of 10668 nodes (resp. 1800 nodes) represented on Figure 14 and was obtained after 10000 explicit steps.

The coupled calculations are initialized by the global Navier-Stokes solution with linear slip boundary conditions and keep the same global mesh for the Navier-Stokes part of the coupled calculation. The local Boltzmann domain is a $0.055 \times 0.005$ square box discretized 


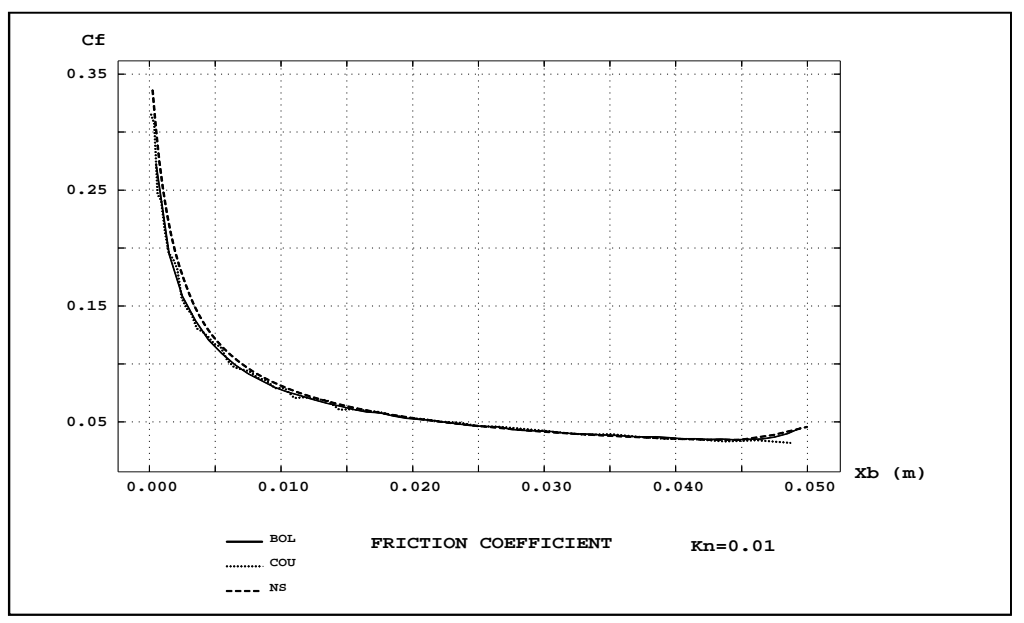

Figure 15: Wall values of the friction coefficient for the three approaches in the dense case $(K n=0.01)$ : Boltzmann, Navier-Stokes and coupled.

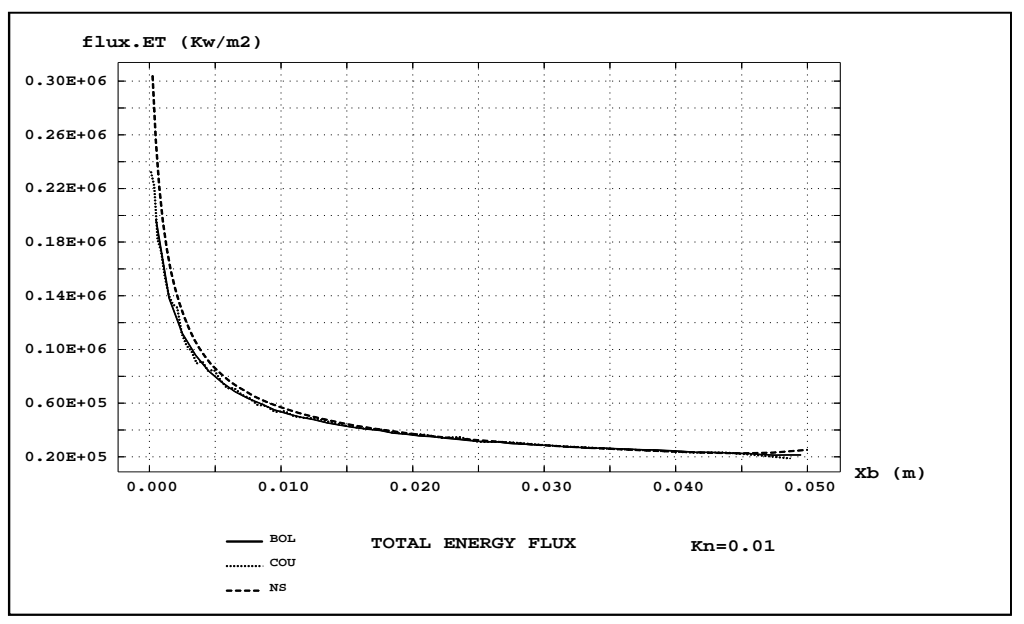

Figure 16: Wall values of the total energy flux for the three approaches in the dense case $(K n=0.01)$ : Boltzmann, Navier-Stokes and coupled.

in 2544 (resp. 2484 ) rectangular cells and using 80000 (resp. 110000 ) particles. We have performed 3 coupling iterations, each iteration consisting of 2000 (resp. 500) time steps for the local Boltzmann problem and of 1000 time steps for the global Navier-Stokes problem. Coupling was realized through a Unix shell script iteratively calling the Boltzmann code and the Navier-Stokes solver.

We present successively the values of the friction coefficient $C_{f}$, of the total energy flux at the wall, the isodensity lines as computed in the local Boltzmann approach, and density or temperature profiles.

In the dense case, all three approaches yield almost identical results. We can nevertheless observe a slight discrepancy between the global Boltzmann and Navier-Stokes solutions both on the energy flux and on the density or temperature profiles. This is very likely due 


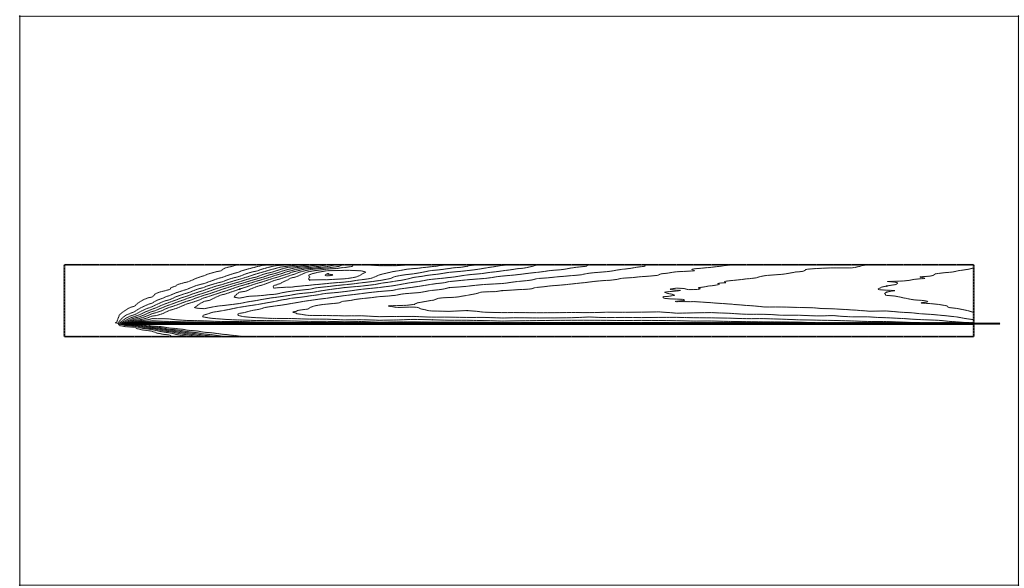

Figure 17: Isodensity lines as predicted by the local Boltzmann model in the dense coupled case $(K n=0.01)$.

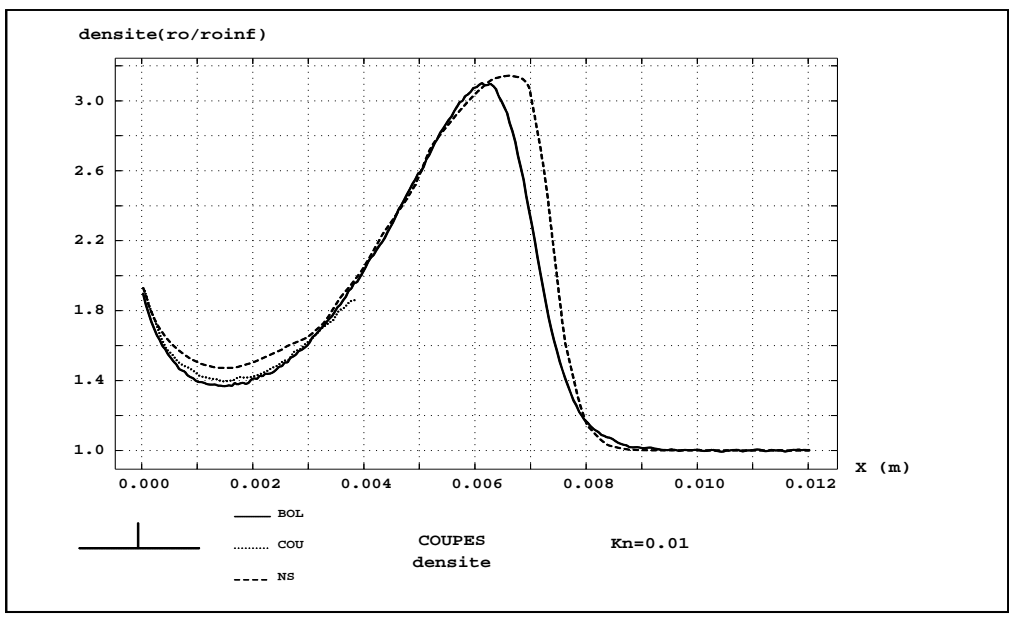

Figure 18: Density profiles for the three approaches in the dense case $(K n=0.01)$ : Boltzmann, Navier-Stokes and coupled. 


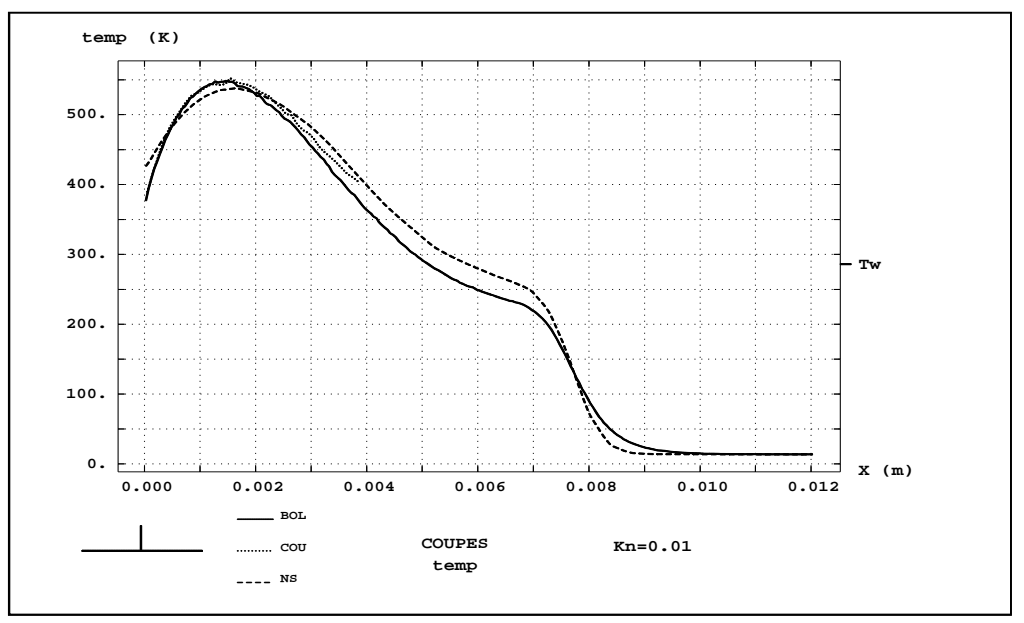

Figure 19: Temperature profiles for the three approaches in the dense case $(K n=0.01)$ : Boltzmann, Navier-Stokes and coupled. Observe the small difference in the temperature jump predicted by the linear slip boundary conditions.

to the physical inaccuracy of the linear slip boundary used for Navier-Stokes. On the other hand, the coupled solution reproduces exactly the behavior of the full kinetic solution.

For a denser case run at Knudsen $K n=0.006$, all three models gave almost identical results. For the more rarefied case however $(K n=0.08)$, Navier-Stokes and Boltzmann simulation yield very different results, and this discrepancy can no longer be recovered by the coupled approach. We clearly see on the different results how the improper Navier-Stokes boundary conditions imposed at the interface affect the behavior of the local Boltzmann solution at the wall after the first fifth of the plate. In such situation, the coupled approach is no longer valid. It could be made more accurate by increasing the size of the local Boltzmann domain, but then the coupled approach is not competitive with respect to a full kinetic simulation.

As a last test, we have rerun the dense calculation with a coupled approach using a local Boltzmann domain which was two times smaller $(0.055 \times 0.0025)$. The local isodensity lines and the wall friction values are represented on Figures 25 and 26 . In this situation, the local domain appears to be a bit too small. The introduction of incoming Maxwellian distributions at the interface slightly perturbs the density next to the interface, and results into a small diminution of the friction coefficient downstream.

\section{Conclusions}

This numerical test performed in this study confirm the validity of the Boltzmann/NavierStokes strategy realized herein by using friction boundary conditions.

We highlighted in this work the convergence of the method and the good agreement of the results obtained by our strategy and those obtained by a direct kinetic simulation for transitional regimes. The advantage of the proposed coupled strategy is to require less computing time and to be applicable in situations where the direct Boltzmann simulation is not possible (because of the lack of memory place and computer power). 


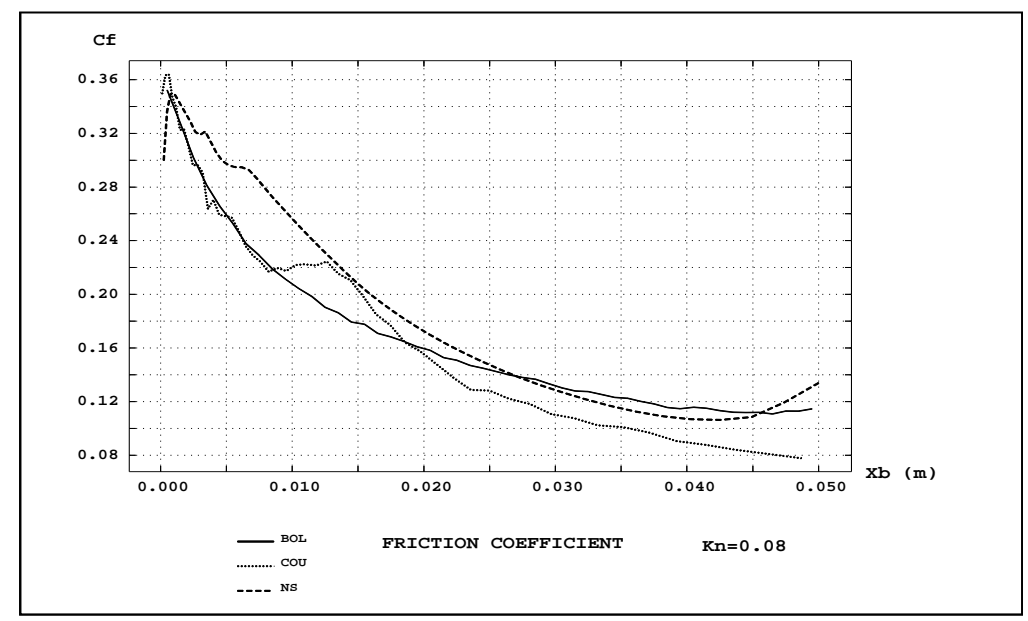

Figure 20: Wall values of the friction coefficient for the three approaches in the rarefied case $(K n=0.08)$ : Boltzmann, Navier-Stokes and coupled.

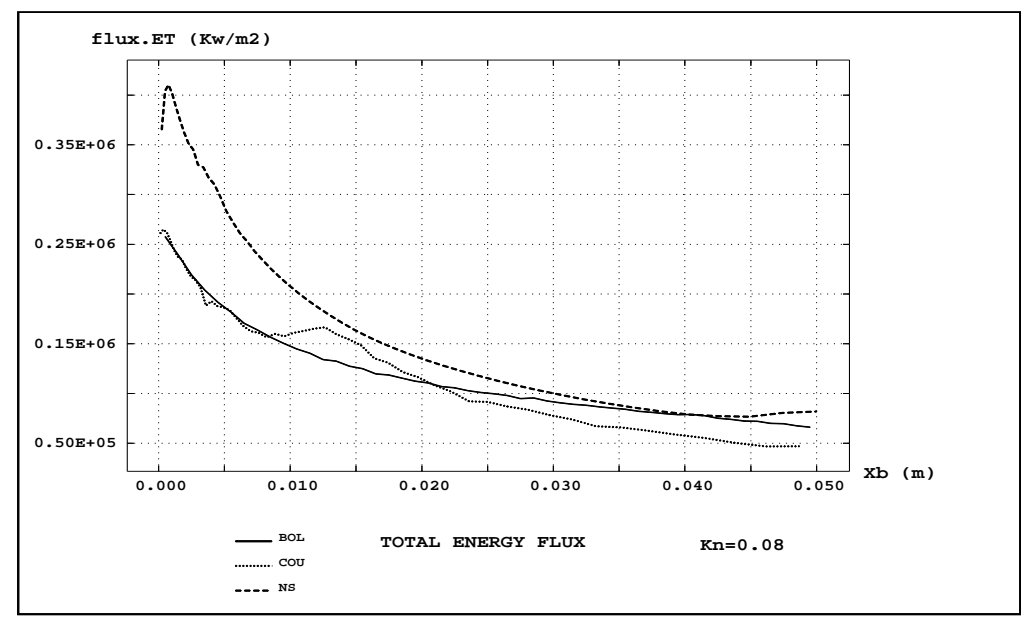

Figure 21: Wall values of the total energy flux for the three approaches in the rarefied case $(K n=0.08)$ : Boltzmann, Navier-Stokes and coupled. 


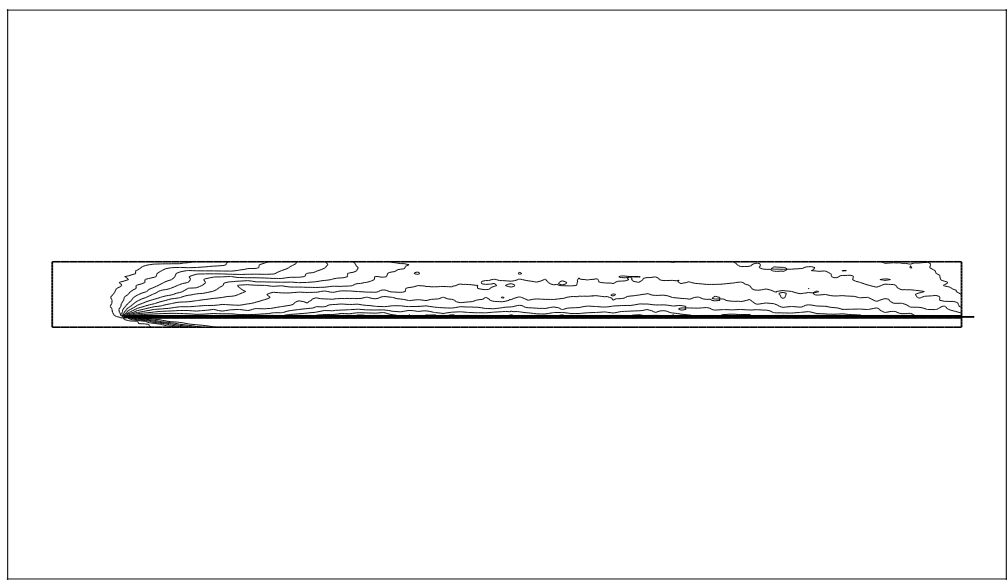

Figure 22: Isodensity lines as predicted by the local Boltzmann model in the rarefied coupled case ( $K n=0.08)$. Observe the influence of the Navier-Stokes solution as soon as the shock crosses the interface.

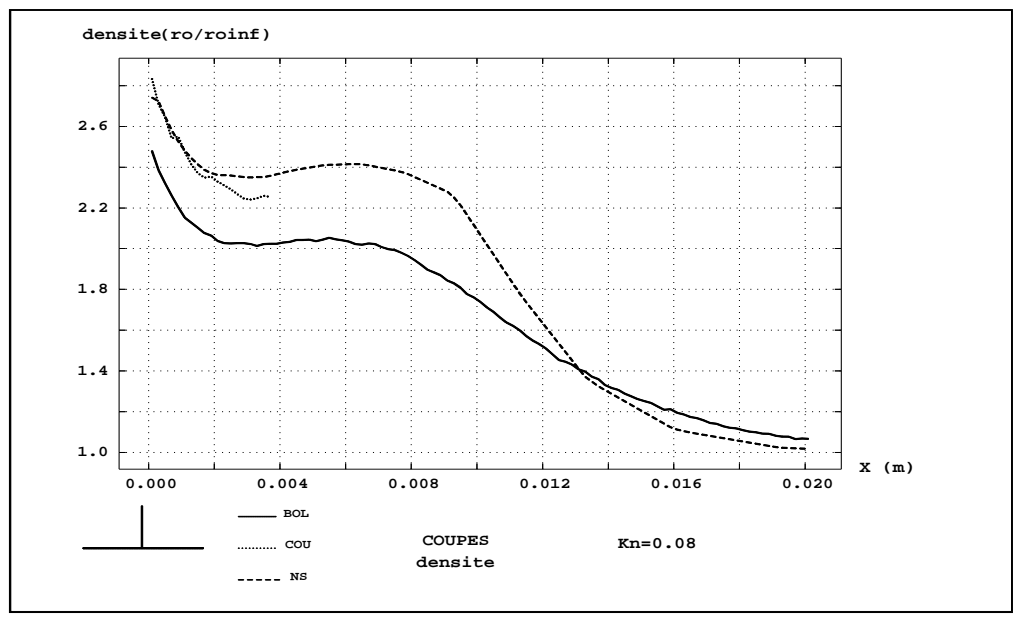

Figure 23: Density profiles for the three approaches in the rarefied case $(K n=0.08)$ : Boltzmann, Navier-Stokes and coupled. 


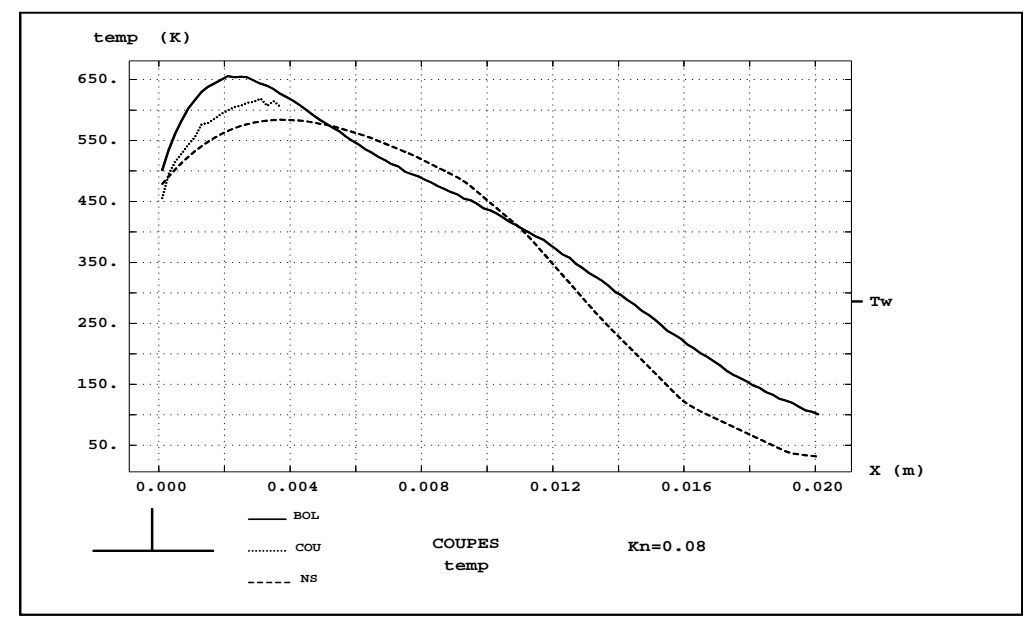

Figure 24: Temperature profiles for the three approaches in the rarefied case $(K n=0.08)$ : Boltzmann, Navier-Stokes and coupled.

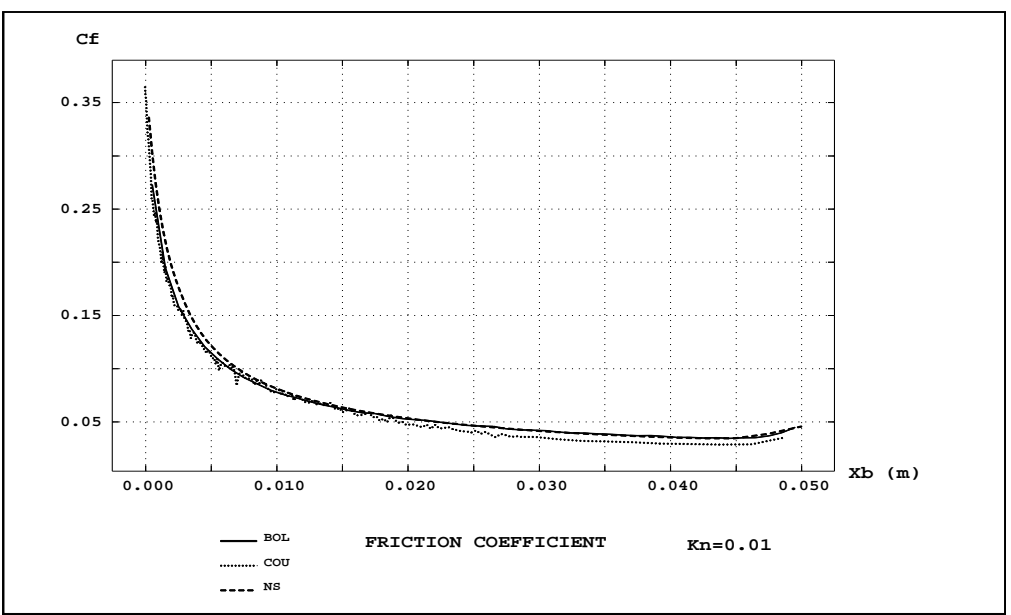

Figure 25: Wall values of the friction coefficient for the three approaches in the dense case with a small local kinetic domain $(K n=0.01)$ : Boltzmann, Navier-Stokes and coupled. Downstream, the coupled friction is a bit smaller than both the Boltzmann and the NavierStokes values. 


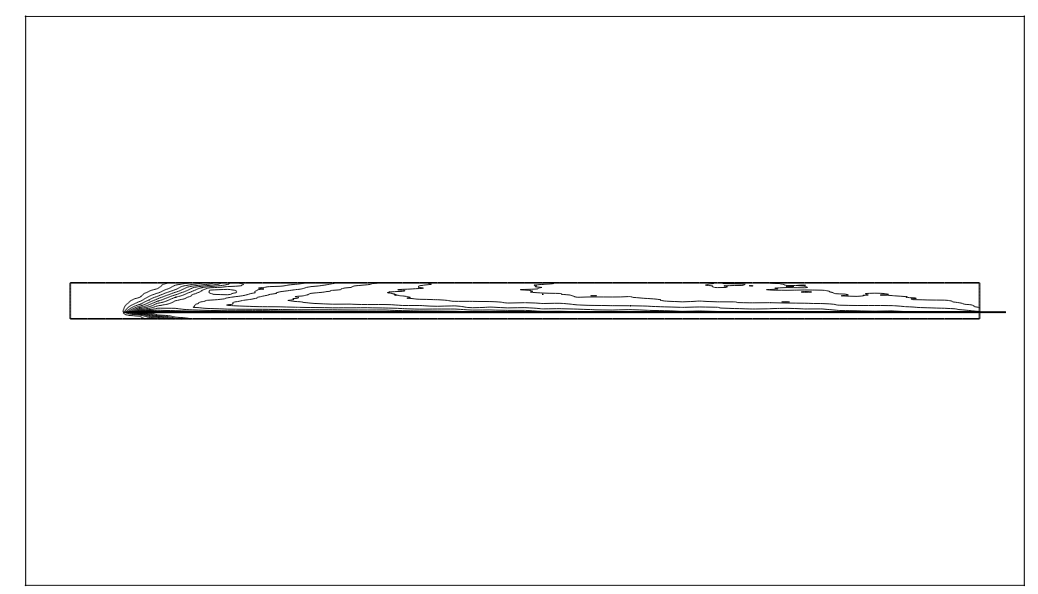

Figure 26: Isodensity lines as predicted by the local Boltzmann model in the dense coupled case $(K n=0.01)$ using a small local kinetic domain.

The coupling strategy allowed also the treatment of experimental situations in the transitional regimes and for two dimensional gas flows. The results obtained are compatible with available experimental results and lead to the determination of a realistic accomodation coefficient at the body.

The difficulties met in this study are of two types :

First the CPU time of the coupling approach is large. It requires more than twice the CPU time needed for a direct Navier-Stokes simulation. However the latter fails for more complex physical situations, while the proposed strategy works well.

Second the Navier-Stokes calculations performed within the coupling strategy are difficult. The convergence is slow, requires more robustness of the slope limiters(we have used monotonic limiters) and the results are sensitive to the mesh used.

Finally, the domain of validity of the coupled approach is hard to identify. When the gas is too dense ( $\left.K n \leq 10^{-} 4\right)$, the coupled approach does not bring any improvement compared to Navier-Stokes models used with linear slip boundary conditions but it validates these linear slip bondary conditions. When the gas is too rarefied $\left(K n \geq 0.510^{-} 1\right)$, the coupling is inaccurate. When the local domain is too small, the assumption of local equilibrium on the incoming distribution slightly perturbs the wall values of friction and heat flux.

In order to circumvent those difficulties, the coupling of Navier-Stokes with Boltzmann on nonoverlapping domains using an adaptive definition of the Boltzmann domain and half fluxes interface matching is presently studied (see [2], [14]).

\section{References}

[1] H. Babovsky. On a simulation scheme for the Boltzmann equation. Math. Meth. in the Appli. Sc. 8, (1986) p. 223-233.

[2] J. F. Bourgat, P. Le Tallec, F. Mallinger, Y. Qiu and M. D. Tidriri, "Numerical coupling of Boltzmann and Navier-Stokes". In proceeding of the sixth I.U.T.A.M. 
(International Union of Theoretical and Applied Mechanics) conference on rarefied flows for reentry problems, Marseille, France September 1992.

[3] J.F. Bourgat, Notice d'utilisation du logiciel BOL2D pour la simulation bidimensionnelle de l'équation de Boltzmann, version 2, INRIA technical report 142, December 1992.

[4] J.F. Bourgat, L. Desvillettes, P. Le Tallec and B. Perthame, Microreversible collisions for polyatomic gases and Boltzmann's theorem, Eur. J. Mech, B/Fluids, 13, n 2, (1994) p. 237-254.

[5] C. Borgnakke, P.S. Larsen, Statistical collision model for Monte-Carlo simulation of polyatomic gas mixtures. J.C.P. 18, 405-420 (1975).

[6] R. Brun, Transport et relaxation dans les écoulements gazeux Masson, Paris (1986).

[7] C. Cercignani, The Boltzmann equation and its applications, Applied Mathematical Sciences $\mathrm{n}^{\circ} 67$, Springer, 1988.

[8] F. Coron, "Applications de la théorie cinétique à l'aoerodynamique hypersonique: une approche mathématique", Thèse, Université de Paris Nord, avril 1989.

[9] R. Gupta, C. Scott and J. Moss, "Slip-boundary equations for multi-component nonequilibrium airflow”. NASA Technical Paper 2452, November 1985.

[10] H. Hollanders and G. Rollin, "Calcul d'écoulements visqueux de gaz parfait par résolution des équations de Navier-Stokes compressibles avec conditions de glissement à la paroi". Rapport ONERA 13/3637 AY, May 1991.

[11] J.C. Lengrand, J.C. Heffner and A. Chpoun, "Rampe de compression en gaz raréfiés," Laboratoire d'Aerothermique, Report RC-90-08, Decembre 1990.

[12] P. Le Tallec and M. D. Tidriri, "Convergence of domain decomposition algorithms with full overlapping for the advection-diffusion problems". Submitted Math. Comp.

[13] P. Le Tallec, M. D. Tidriri, "Kinetic upgrade of Navier-Stokes equations for transitional flows." Hermes Contract Report, December 1993.

[14] P. Le Tallec and F. Mallinger, Couplage Boltzmann Navier-Stokes, in Proceedings of the 57th meeting between Theoretical Physics and Mathematics, Université Louis Pasteur, December 1993.

[15] M. Mallet, "A finite element method for computational fluid dynamics", Ph.D. Thesis, Stanford University, 1985

[16] Ph. Rostand, B. Stoufflet, " Finite volume Galerkin methods for viscous gas dynamics", Rapport de recherche INRIA no 863, Juillet 1988. 
[17] Y. Saad and M. H. Schultz, "GMRES: A Generalized Minimal Residual Algorithm for Solving Nonsymmetric Linear Systems", SIAM J. Sci. Stat. Comput. 7, 856 (1986).

[18] F. Shakib, "Finite element analysis of the compressible Euler and Navier-Stokes equations", Ph.D. Thesis, Stanford University, 1988

[19] M. D. Tidriri, "Couplage d'approximations et de modèles de types différents dans le calcul d'écoulements externes", thèse, Université de Paris IX, May 1992.

[20] M. D. Tidriri, "Domain decomposition for compressible Navier-Stokes equations with different discretizations and formulations." Accepted for publication in J. Comp. Phy.

[21] M. D. Tidriri, "Domain decomposition for incompatible nonlinear models", INRIA Research Report 2435, Dec 94.

[22] W.G. Vicenti and C. H. Kruger Jr., "Introduction to Physical Gas Dynamics", Wiley, 1967.

[23] X. Zhong, R.W. MacCormack and D.R. Chapman, "Stabilization of the Burnett Equations and Application to Hypersonic Flows", AIAA Journal, 31,6,1993. 
Unité de recherche INRIA Lorraine, Technopôle de Nancy-Brabois, Campus scientifique, 615 rue du Jardin Botanique, BP 101, 54600 VILLERS LÈS NANCY

Unité de recherche INRIA Rennes, Irisa, Campus universitaire de Beaulieu, 35042 RENNES Cedex

Unité de recherche INRIA Rhône-Alpes, 46 avenue Félix Viallet, 38031 GRENOBLE Cedex 1

Unité de recherche INRIA Rocquencourt, Domaine de Voluceau, Rocquencourt, BP 105, 78153 LE CHESNAY Cedex

Unité de recherche INRIA Sophia-Antipolis, 2004 route des Lucioles, BP 93, 06902 SOPHIA-ANTIPOLIS Cedex

\section{Éditeur}

INRIA, Domaine de Voluceau, Rocquencourt, BP 105, 78153 LE CHESNAY Cedex (France)

ISSN 0249-6399 